\title{
Networking Seifert surgeries on knots, III
}

\author{
ARNAUD DERUELLE \\ KATURA MIYAZAKI \\ KIMIHIKO MOTEGI
}

\begin{abstract}
How do Seifert surgeries on hyperbolic knots arise from those on torus knots? We approach this question from a networking viewpoint introduced in [9]. The Seifert surgery network is a 1-dimensional complex whose vertices correspond to Seifert surgeries; two vertices are connected by an edge if one Seifert surgery is obtained from the other by a single twist along a trivial knot called a seiferter or along an annulus cobounded by seiferters. Successive twists along a "hyperbolic seiferter" or a "hyperbolic annular pair" produce infinitely many Seifert surgeries on hyperbolic knots. In this paper, we investigate Seifert surgeries on torus knots that have hyperbolic seiferters or hyperbolic annular pairs, and obtain results suggesting that such surgeries are restricted.
\end{abstract}

$57 \mathrm{M} 25 ; 57 \mathrm{M} 50,57 \mathrm{~N} 10$

Dedicated to Sadayoshi Kojima on the occasion of his $60^{\text {th }}$ birthday

\section{Introduction}

How do Seifert surgeries on hyperbolic knots arise from those on torus knots? In [9] we formulate this question from a viewpoint of the Seifert surgery network. Let us recall some basic notions given in [9] and an example illustrating our idea. A pair $(K, m)$ of a knot $K$ in $S^{3}$ and an integer $m$ is a Seifert surgery if the result $K(m)$ of $m$-Dehn surgery on $K$ has a Seifert fibration; we allow the fibration to be degenerate, ie to contain an exceptional fiber of index 0 as a degenerate fiber. It is shown in [9, Proposition 2.8] that if $K(m)$ admits a degenerate Seifert fibration, then it is either a lens space or a connected sum of two lens spaces. In the latter case, Greene [14] recently showed that $K$ is a torus knot or a cable of a torus knot.

Definition 1.1 (Seiferter) Let $(K, m)$ be a Seifert surgery. A knot $c$ in $S^{3}-N(K)$ is called a seiferter for $(K, m)$ if $c$ satisfies (1) and (2) below:

(1) $c$ is a trivial knot in $S^{3}$.

(2) $c$ becomes a fiber in a Seifert fibration of $K(m)$. 
We also consider pairs of seiferters.

Definition 1.2 (Annular pair of seiferters) Let $c_{1}, c_{2}$ be seiferters for a Seifert surgery $(K, m)$. We call $\left\{c_{1}, c_{2}\right\}$ a pair of seiferters if $c_{1}$ and $c_{2}$ simultaneously become fibers in a Seifert fibration of $K(m)$. A pair of seiferters $\left\{c_{1}, c_{2}\right\}$ is called a Hopf pair if $c_{1} \cup c_{2}$ is a Hopf link in $S^{3}$. A pair of seiferters $\left\{c_{1}, c_{2}\right\}$ is called an annular pair of seiferters (or annular pair for short) if $c_{1}$ and $c_{2}$ cobound an annulus in $S^{3}$.

For a Seifert surgery $(K, m)$ with a seiferter $c$, let $K_{p}$ and $m_{p}$ be the images of $K$ and $m$ under a $p$-twist along $c$, respectively. Then, $\left(K_{p}, m_{p}\right)$ remains a Seifert surgery for any integer $p$, and (the image of) $c$ is also a seiferter for $\left(K_{p}, m_{p}\right)$ [9, Proposition 2.6]. Similarly, if $(K, m)$ has an annular pair $\left\{c_{1}, c_{2}\right\}$, then under twisting along the annulus cobounded by $c_{1}, c_{2}$, we obtain a new Seifert surgery for which (the image of) $\left\{c_{1}, c_{2}\right\}$ remains an annular pair [9, Proposition 2.33(1)]. We call a twist along an annulus cobounded by $c_{1} \cup c_{2}$ a twist along an annular pair of seiferters. We say that a seiferter $c$ (respectively an annular pair $\left.\left\{c_{1}, c_{2}\right\}\right)$ for a Seifert surgery $(K, m)$ is hyperbolic if $S^{3}-K \cup c$ (respectively $S^{3}-K \cup c_{1} \cup c_{2}$ ) admits a complete, hyperbolic metric with finite volume.

Remark 1.3 Suppose that a seiferter $c$ for $(K, m)$ bounds a disk in $S^{3}-K$. Since no twist along $c$ changes $(K, m)$, we call $c$ irrelevant. We do not regard an irrelevant seiferter as a seiferter. However, for pairs of seiferters $\left\{c_{1}, c_{2}\right\}$ we allow $c_{i}$ to be an irrelevant seiferter. Let $\left\{c_{1}, c_{2}\right\}$ be an annular pair for $(K, m)$. If either $c_{1}$ and $c_{2}$ cobound an annulus disjoint from $K$ or there is a $2-$ sphere in $S^{3}$ separating $c_{i}$ and $c_{j} \cup K$, then twists along $\left\{c_{1}, c_{2}\right\}$ do not change $(K, m)$ or have the same effect on $K$ as twists along $c_{j}$. We thus call such an annular pair irrelevant, and exclude it from annular pairs of seiferters. Note that if $S^{3}-K \cup c_{1} \cup c_{2}$ is hyperbolic, then $\left\{c_{1}, c_{2}\right\}$ is not irrelevant.

Regard each Seifert surgery as a vertex, and connect two vertices by an edge if one is obtained from the other by a single twist along a seiferter or an annular pair of seiferters. We then obtain a 1-dimensional complex, called the Seifert surgery network.

Let us take a look at seiferters for Seifert surgeries on torus knots $T_{p, q}$. Throughout this paper we assume, without loss of generality, that $|p|>q \geq 1$, and denote a trivial knot $T_{p, 1}$ by $O$.

Example 1.4 (The subcomplex $\mathcal{T}$ ) Since the exterior of a torus knot $T_{p, q}$ is a Seifert fiber space, $\left(T_{p, q}, m\right)$ is a Seifert surgery for any integer $m$. Let $s_{p}, s_{q}$ be exceptional fibers in the Seifert fibration of the exterior of $T_{p, q}$ with indices $|p|, q$, respectively, 
and $c_{\mu}$ a meridian of $T_{p, q}$; see Figure 1.1. Then $s_{p}$ and $s_{q}$ remain exceptional fibers in $T_{p, q}(m)$. Note that $c_{\mu}$ is isotopic in $T_{p, q}(m)$ to the core of the filled solid torus, which is a fiber of index $|p q-m|$ and in particular a degenerate fiber in $T_{p, q}(p q)$. Hence, the trivial knots $s_{p}, s_{q}, c_{\mu}$ are seiferters for $\left(T_{p, q}, m\right)$ for any integer $m$, and called basic seiferters for $\left(T_{p, q}, m\right)$. We denote by $\mathcal{T}$ the subcomplex whose vertices are Seifert surgeries on torus knots and whose edges correspond to basic seiferters.

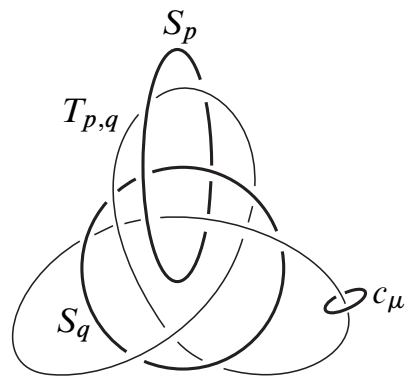

Figure 1.1: Basic Seiferters

The following example motivates us to consider the Seifert surgery network.

Example 1.5 (1) The meridian $c_{\mu}$ for $T_{-3,2}$ is a seiferter for all $\left(T_{-3,2}, m\right)$ $(m \in \mathbb{Z})$. Twisting along $c_{\mu}$ yields the horizontal line in Figure 1.2, which consists of all the integral Seifert surgeries on $T_{-3,2}$.

(2) The trivial knot $c \subset S^{3}-T_{-3,2}$ in Figure 1.2 is a seiferter for the Seifert surgery $\left(T_{-3,2},-2\right)$ (Section 2, Figure 2.2). A (-2)-twist of $T_{-3,2}$ along $c$ yields the figure-eight knot. Since the linking number between $c$ and $T_{-3,2}$ is zero, the surgery slope -2 does not change under the twisting. Thus we obtain the right vertical line in Figure 1.2.

(3) The trivial knot $c^{\prime} \subset S^{3}-T_{-3,2}$ in Figure 1.2 is a seiferter for $\left(T_{-3,2},-7\right)$ [9, Example 2.21(2)]. A 1 -twist of $T_{-3,2}$ along $c^{\prime}$ yields the $(-2,3,7)$ pretzel knot $P(-2,3,7)$; see the authors [7]. Since the linking number between $c^{\prime}$ and $T_{-3,2}$ is 5 , the surgery slope changes from -7 to $-7+5^{2}=18$. We thus obtain the lens surgery $(P(-2,3,7), 18)$ first found by Fintushel and Stern [12]. This gives the left vertical line in Figure 1.2.

A path from $(K, m)$ to $\left(K^{\prime}, m^{\prime}\right) \in \mathcal{T}$ in the network shows that the Seifert surgery $(K, m)$ is obtained from the $m^{\prime}$-surgery on the torus knot $K^{\prime}$ by a sequence of twists along seiferters or annular pairs. For example, vertical paths in Figure 1.2 from (figure-eight knot, -2$)$ and $(P(-2,3,7), 18)$ to vertices in $\mathcal{T}$ explain how these surgeries arise from surgeries on a trefoil knot. The authors $[8 ; 9]$ and the authors with 


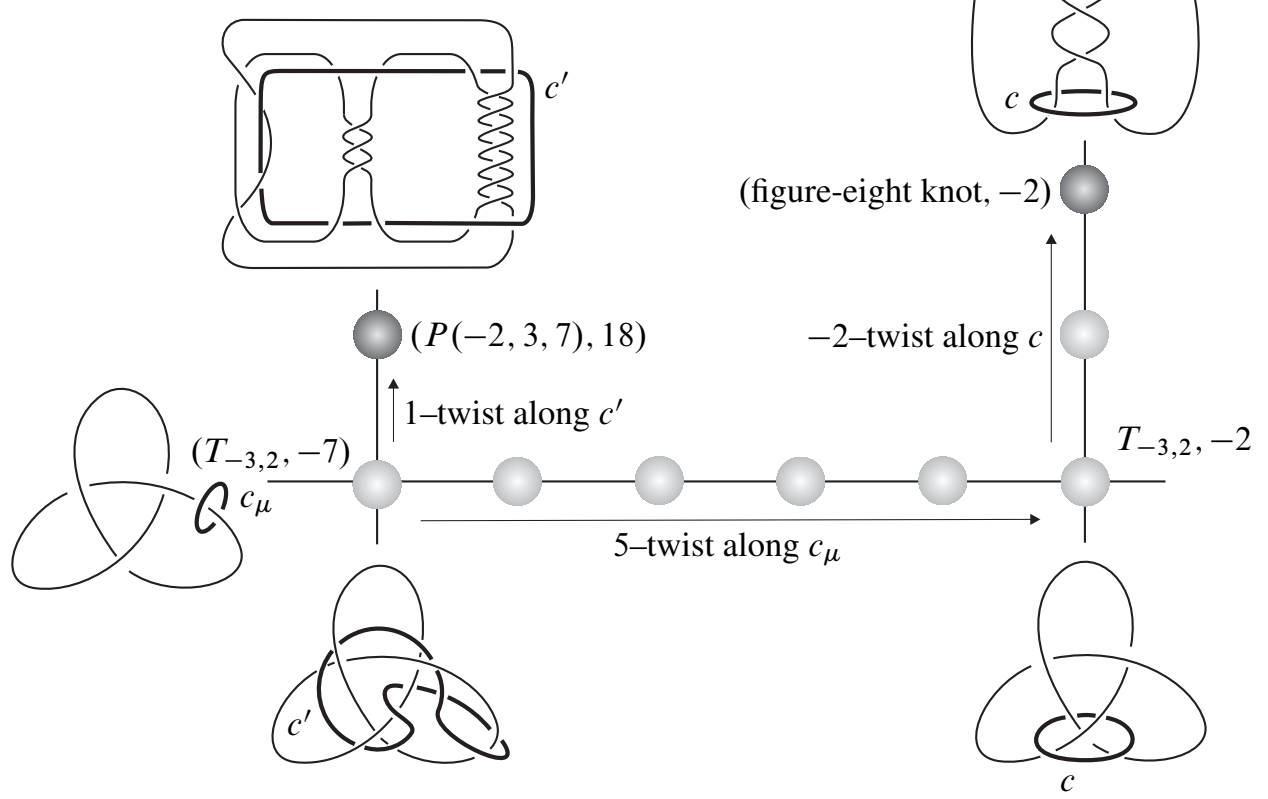

Figure 1.2: Seifert surgery network

Eudave-Muñoz [6] find paths from various known Seifert surgeries to vertices in $\mathcal{T}$; the list includes Seifert surgeries on graph knots, Berge's lens surgeries [3], and Seifert surgeries constructed by using Montesinos' trick [10; 11].

In the present paper, we explore Seifert surgeries on torus knots which have edges going out of $\mathcal{T}$, and try to classify such surgeries. We focus on Seifert surgeries on torus knots which have hyperbolic seiferters or hyperbolic annular pairs. By Thurston's hyperbolic Dehn surgery theorem (see Thurston [28; 29], Benedetti and Petronio [2], Petronio and Porti [25] and Boileau and Porti [4]), if $\left(T_{p, q}, m\right)$ has a hyperbolic seiferter (respectively a hyperbolic annular pair), then all but finitely many vertices of the 1-complex generated by successive twists along the seiferter (respectively the annular pair) are Seifert surgeries on hyperbolic knots. Hence, we call $\left(T_{p, q}, m\right)$ with a hyperbolic seiferter or a hyperbolic annular pair a spreader. Previously known examples of spreaders $[6 ; 7 ; 8 ; 9]$ have specific patterns and lead us to the following conjecture.

Conjecture 1.6 If $\left(T_{p, q}, m\right)$ is a spreader, then $q=1,2$, or $m=p q, p q \pm 1$.

In Section 2, we review the definition of $m$-moves introduced in [9], which are in fact Kirby calculus handle-slides over $m$-framed knots. A trivial knot obtained from a 
seiferter for $(K, m)$ by a sequence of $m$-moves is also a seiferter for $(K, m)$ if $K$ is nontrivial. In Sections 3 and 4 , we exploit $m$-moves to find seiferters for $\left(T_{p, q}, m\right)$ where $q=1,2$. Theorems 3.1 and 4.1 imply the following.

Theorem 1.7 For each integer $m,\left(T_{p, 1}, m\right)=(O, m)$ and $\left(T_{p, 2}, m\right)$ are spreaders. In particular, $(O, m)$ has infinitely many hyperbolic annular pairs as well as infinitely many hyperbolic seiferters.

Regarding seiferters for $\left(T_{p, q}, m\right)$ where $q \geq 3$, we consider two cases according to whether $T_{p, q}(m)$ has a unique Seifert fibration up to isotopy or not: the case when $|m-p q| \geq 2$ and the case when $m=p q, p q \pm 1$. In the latter case, we prove the theorem below, which follows from Propositions 5.1, 5.4 and 5.5.

Theorem 1.8 Both $\left(T_{p, q}, p q\right)(q \geq 2,(p, q) \neq( \pm 3,2))$ and $\left(T_{2 n \pm 1, n}, n(2 n \pm 1)-1\right)$ $(n \geq 2)$ have a hyperbolic seiferter which cannot be obtained from basic seiferters or a regular fiber of the exterior of the torus knot by any sequence of $m$-moves.

Conjecture 1.6 above implies that if $q \geq 3$ and $m \neq p q, p q \pm 1,\left(T_{p, q}, m\right)$ has no hyperbolic seiferters. Theorem 1.9 below shows the difficulty of obtaining such a hyperbolic seiferter.

Theorem 1.9 Suppose that $q \geq 3$ and $m \neq p q, p q \pm 1$ (ie $T_{p, q}(m)$ is not a connected sum of lens spaces, a lens space, or a prism manifold). Then every seiferter for $\left(T_{p, q}, m\right)$ is obtained from a basic seiferter or a regular fiber of $S^{3}-N\left(T_{p, q}\right)$ by a sequence of $m$-moves (Proposition 2.2). However, to obtain a hyperbolic seiferter for $\left(T_{p, q}, m\right)$ in such a manner we need to apply $m$-moves at least twice (Corollary 6.8(2)).

We close the introduction with the following question.

Question 1.10 Does every lens surgery $\left(T_{p, q}, p q \pm 1\right)$ have a hyperbolic seiferter?

Acknowledgments We would like to thank Mario Eudave-Muñoz, Cameron Gordon, John Luecke, Daniel Matignon, José María Montesinos, Kunio Murasugi, Kouki Taniyama, Masakazu Teragaito, and Akira Yasuhara for useful discussions and encouragements. We would also like to thank the referee for careful reading and useful comments.

The third author has been partially supported by JSPS Grants-in-Aid for Scientific Research (C) (number 17540097 and number 21540098), The Ministry of Education, Culture, Sports, Science and Technology, Japan and Joint Research Grant of Institute of Natural Sciences at Nihon University for 2012. 


\section{Seiferters for torus knots and Seifert fibrations of torus knot spaces}

Definition 2.1 ( $m$-move) Let $K$ be a knot in $S^{3}$, and $c$ a knot in $S^{3}-N(K)$. Take a simple closed curve $\alpha_{m}$ on $\partial N(K)$ representing a slope $m$. Let $b$ be a band in $S^{3}-$ int $N(K)$ connecting $\alpha_{m}$ and $c$, and let $b \cap \alpha_{m}=\tau_{\alpha_{m}}, b \cap c=\tau_{c}$. We set $\tau_{\alpha_{m}}^{\prime}=\alpha_{m}-$ int $\tau_{\alpha_{m}}$ and $\tau_{c}^{\prime}=c-$ int $\tau_{c}$. Then the band connected sum $c \natural_{b} \alpha_{m}=\tau_{c}^{\prime} \cup\left(\partial b-\operatorname{int}\left(\tau_{c} \cup \tau_{\alpha_{m}}\right)\right) \cup \tau_{\alpha_{m}}^{\prime}$ is a knot in $S^{3}-\operatorname{int} N(K)$. Pushing $c \natural_{b} \alpha_{m}$ away from $\partial N(K)$, we obtain a knot $c^{\prime}$ in $S^{3}-N(K)$; see Figure 2.1. We say that $c^{\prime}$ is obtained from $c$ by an $m$-move using the band $b$.

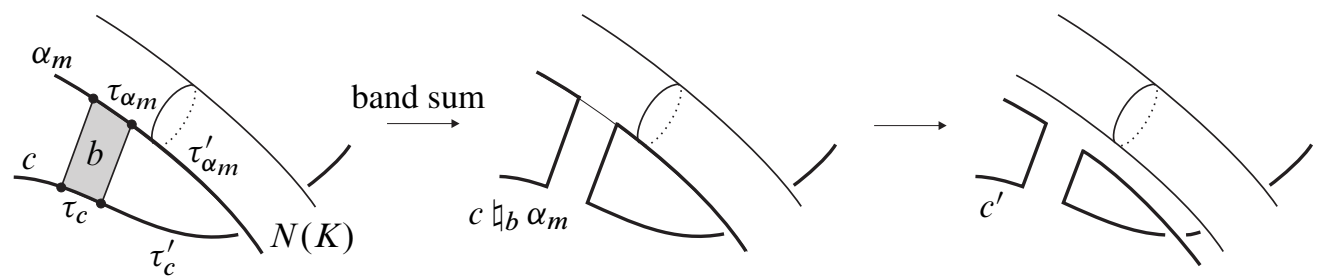

Figure 2.1: $m$-move

Let $K$ be a knot in $S^{3}$, and $c_{1}, c_{2}$ knots in $S^{3}-N(K)$. Assume that $c_{2}$ is obtained from $c_{1}$ after a finite sequence of $m$-moves and isotopies in $S^{3}-\operatorname{int} N(K)$. We then say that $c_{2}$ is $m$-equivalent to $c_{1}$. Note that $c_{2}$ is isotopic to $c_{1}$ in the surgered manifold $K(m)$ [9, Proposition 2.19(1)]. Hence, if $(K, m)$ is a Seifert surgery, $c_{1}$ is a seiferter for $(K, m)$, and $c_{2}$ is a trivial knot, then $c_{2}$ is a possibly irrelevant seiferter for $(K, m)$. Proposition 2.19(3) in [9] shows that $c_{2}$ is not irrelevant if $K$ is a nontrivial knot. Figure 2.2 illustrates how an $m$-move works, where $K=T_{-3,2}$, $m=-2, c_{1}=s_{-3}$. It follows that $c_{2}$ is a seiferter for $\left(T_{-3,2},-2\right)$. See Section 3 for $m-$ moves of annular pairs of seiferters.
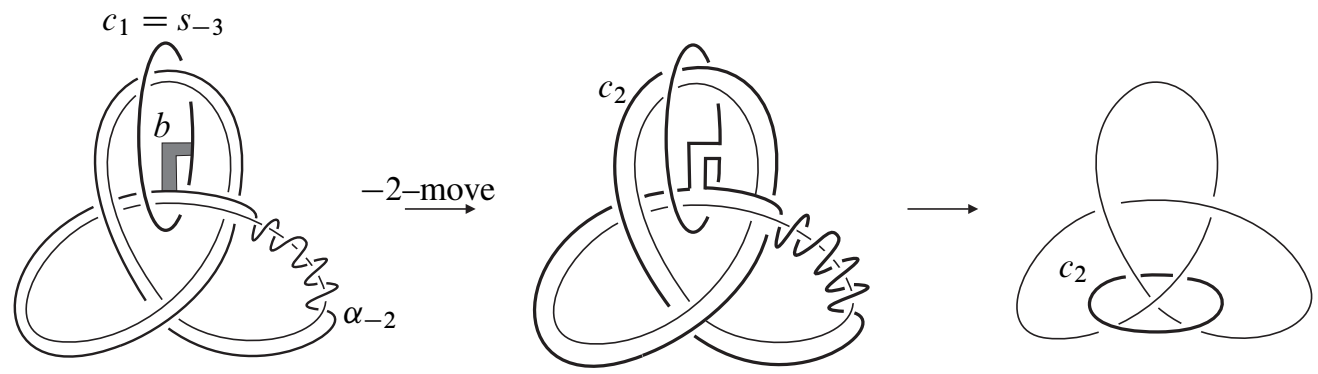

Figure 2.2: $m$-move; $m=-2, c_{2}$ is a seiferter for $\left(T_{-2,3},-2\right)$ 
Most seiferters for $\left(T_{p, q}, m\right)$ are $m$-equivalent to basic seiferters or regular fibers of Seifert fibrations of $S^{3}-$ int $N\left(T_{p, q}\right)$. Precise statements are as follows.

Proposition 2.2 Let $T_{p, q}$ be a nontrivial torus knot, and $c$ a seiferter for $\left(T_{p, q}, m\right)$, where $m \neq p q$.

(1) Suppose that $c$ is an exceptional fiber in some Seifert fibration of $T_{p, q}(m)$. If $T_{p, q}(m)$ is a lens space, we assume that the base surface is $S^{2}$. Then $c$ is $m$-equivalent to a basic seiferter $s_{p}, s_{q}$ or $c_{\mu}$.

(2) Suppose that $c$ is a regular fiber in some Seifert fibration of $T_{p, q}(m)$. If $T_{p, q}(m)$ is neither a lens space nor a prism manifold, then $c$ is $m$-equivalent to a regular fiber in $S^{3}-N\left(T_{p, q}\right)$.

Proof of Proposition 2.2 We denote by $\mathcal{F}$ a Seifert fibration on $T_{p, q}(m)$ in which $c$ is an exceptional fiber or a regular fiber.

Case $1 T_{p, q}(m)$ is not a lens space.

By [9, Proposition 2.8], if $T_{p, q}(m)$ admits a degenerate Seifert fibration, then it is either a lens space or a connected sum of two lens spaces. It follows that $\mathcal{F}$ is a nondegenerate Seifert fibration. Let $\mathcal{F}_{0}$ be a natural extension of the Seifert fibration of $S^{3}-$ int $N\left(T_{p, q}\right)$ over $T_{p, q}(m)$. The base space of $\mathcal{F}_{0}$ is the $2-$ sphere, and its exceptional fibers are $s_{p}, s_{q}$ and a core of the filled solid torus, whose indices are $|p|, q$ and $|p q-m|$, respectively. We note that $c_{\mu}$ is isotopic in $T_{p, q}(m)$ to the third exceptional fiber of $\mathcal{F}_{0}$. Let $t$ be a regular fiber of $\mathcal{F}_{0} \mid\left(S^{3}-N\left(T_{p, q}\right)\right)$.

Subcase $1 T_{p, q}(m)$ is not a prism manifold.

It then follows from Jiang, Wang and $\mathrm{Wu}$ [20, Corollary 3.12] that two Seifert fibrations $\mathcal{F}$ and $\mathcal{F}_{0}$ are isotopic. Hence, if $c$ is an exceptional fiber in $T_{p, q}(m)$, then $c$ is isotopic to one of $s_{p}, s_{q}$ and $c_{\mu}$ in $T_{p, q}(m)$, and thus $m$-equivalent to a basic seiferter $s_{p}, s_{q}$ or $c_{\mu}$. Similarly, if $c$ is a regular fiber in $T_{p, q}(m)$, then $c$ is isotopic to $t$ in $T_{p, q}(m)$ and thus $m$-equivalent to the regular fiber $t$ [9, Proposition 2.19(1)].

Subcase $2 T_{p, q}(m)$ is a prism manifold and $c$ is an exceptional fiber.

A Seifert fibration of a prism manifold is either over $S^{2}$ with three exceptional fibers of indices 2, 2, $x$ or over $\mathbb{R} P^{2}$ with at most one exceptional fiber; see Jaco [19, VI.16(b)]. Hence, $\mathcal{F}_{0}$ is a Seifert fibration over the base orbifold $S^{2}(2,2, x)$ for some odd integer $x(\geq 3)$. Now let us show that $T_{p, q}(m)$ has a Seifert fibration over $S^{2}$ with $c$ an exceptional fiber even if the base space of $\mathcal{F}$ is not $S^{2}$. Assume that $\mathcal{F}$ is a Seifert fibration over $\mathbb{R} P^{2}$; then $\mathcal{F} \mid\left(T_{p, q}(m)-\right.$ int $\left.N(c)\right)$ is a Seifert fibration over the Möbius band 
with no exceptional fiber. Hence $T_{p, q}(m)-$ int $N(c)$ admits a Seifert fibration over the disk with two exceptional fibers of indices 2, 2. Extending this fibration over $T_{p, q}(m)$, we obtain a Seifert fibration over $S^{2}$ with $c$ an exceptional fiber, as claimed. For simplicity, denote the new Seifert fibration by the same symbol $\mathcal{F}$. Then, $\mathcal{F}$ is a Seifert fibration over the base orbifold $S^{2}\left(2,2, x^{\prime}\right)$ for some odd integer $x^{\prime}(\geq 3)$. Since a regular fiber of $\mathcal{F}$ (respectively $\mathcal{F}_{0}$ ) generates the center of $\pi_{1}\left(T_{p, q}(m)\right)$, the quotient of $\pi_{1}\left(T_{p, q}(m)\right)$ by its center is the dihedral group of order $2 x^{\prime}$ (respectively $\left.2 x\right)$. It follows that $x=x^{\prime}$.

Claim 2.3 There exists an orientation preserving homeomorphism $f$ of $T_{p, q}(m)$ which carries fibers of $\mathcal{F}$ to fibers of $\mathcal{F}_{0}$.

Proof of Claim 2.3 We denote the normalized Seifert invariant of $\mathcal{F}$ by $\left(b, \frac{1}{2}, \frac{1}{2}, \frac{y}{x}\right)$ $(b \in \mathbb{Z}, 0<y<x)$, and that of $\mathcal{F}_{0}$ by $\left(b^{\prime}, \frac{1}{2}, \frac{1}{2}, \frac{y^{\prime}}{x}\right)\left(b^{\prime} \in \mathbb{Z}, 0<y^{\prime}<x\right)$. Note that the order of $H_{1}\left(T_{p, q}(m)\right)$ is given by $4|(b+1) x+y|=4\left|\left(b^{\prime}+1\right) x+y^{\prime}\right|$. Hence we have $b=b^{\prime}, y=y^{\prime}$ or $b+b^{\prime}=-3, x=y+y^{\prime}$. In the former case, we have an orientation preserving homeomorphism of $T_{p, q}(m)$ which carries fibers of $\mathcal{F}$ to those of $\mathcal{F}_{0}$ as desired; see Orlik [24], Neumann and Raymond [22] and Hatcher [15]. We show that the latter does not occur. If we have the latter case, then $\left(b^{\prime}, \frac{1}{2}, \frac{1}{2}, \frac{y^{\prime}}{x}\right)=\left(-b-3, \frac{1}{2}, \frac{1}{2}, \frac{x-y}{x}\right)$. On the other hand, $-T_{p, q}(m)\left(T_{p, q}(m)\right.$ with orientation reversed) has a Seifert invariant $\left(-b,-\frac{1}{2},-\frac{1}{2},-\frac{y}{x}\right)$, which is normalized to $\left(-b-3, \frac{1}{2}, \frac{1}{2}, \frac{x-y}{x}\right)$. Thus we have an orientation preserving homeomorphism from $-T_{p, q}(m)$ to $T_{p, q}(m)[15 ; 22 ; 24]$, ie $T_{p, q}(m)$ admits an orientation reversing homeomorphism. This contradicts the fact that a prism manifold has no orientation reversing homeomorphism; see Asano [1;22, 8.4] and Rubinstein [26].

Then, [20, Lemma 3.5] implies that $f$ is isotopic to a homeomorphism preserving $\mathcal{F}$. This implies that $\mathcal{F}_{0}$ is isotopic to $\mathcal{F}$. Hence just as in Subcase 1, the exceptional fiber $c$ is $m$-equivalent to one of $s_{p}, s_{q}$ and $c_{\mu}$.

Case $2 T_{p, q}(m)$ is a lens space, and $c$ is an exceptional fiber.

Then $T_{p, q}(m)$ has a natural Seifert fibration over $S^{2}$ in which $s_{p}$ and $s_{q}$ are exceptional fibers of indices $|p|, q$. Note also that $s_{p}$ and $s_{q}$ give a genus-one Heegaard splitting $T_{p, q}(m)=V \cup W$ of the lens space $T_{p, q}(m) ; s_{p}$ and $s_{q}$ are cores of the solid tori $V$ and $W$. We recall that the base space of the Seifert fibration $\mathcal{F}$ is $S^{2}$ from the assumption of Proposition 2.2(1). Then, $\mathcal{F}$ also gives a genus-one Heegaard splitting $T_{p, q}(m)=V^{\prime} \cup W^{\prime}$ such that the exceptional fiber $c$ in $\mathcal{F}$ is a core of $V^{\prime}$. Since a genus-one Heegaard splitting is unique up to isotopy by Bonahon and Otal [5] and Hodgson and Rubinstein [17], $c$ is isotopic to $s_{p}$ or $s_{q}$ in $T_{p, q}(m)$. Proposition 2.19(1) in [9] thus shows that $c$ is $m$-equivalent to a basic seiferter $s_{p}$ or $s_{q}$ as desired. 
Remark 2.4 Assumptions in Proposition 2.2 are necessary.

(1) As we will see in Proposition 5.1, each $\left(T_{p, q}, p q\right)$ where $(p, q) \neq( \pm 3,2)$ has a seiferter which is not $p q$-equivalent to any basic seiferter nor a regular fiber of $S^{3}-N\left(T_{p, q}\right)$.

(2) If $T_{p, q}(m)$ is a prism manifold (ie $q=2$ and $m=2 p \pm 2$ ), then there exists a seiferter $c$ for $\left(T_{p, q}, m\right)$ which is a regular fiber in a Seifert fibration over the projective plane [9, Corollary 3.15(6)]. Then $c$ is not $m$-equivalent to a regular fiber of $S^{3}-N\left(T_{p, q}\right)$.

(3) Propositions 5.4 and 5.5 show that for some lens surgeries $\left(T_{p, q}, m\right)(m=p q \pm 1)$, there exist seiferters which are not $m$-equivalent to any basic seiferters nor regular fibers of $S^{3}-N\left(T_{p, q}\right)$.

\section{Annular pairs of seiferters for $(O, m)$}

Let $\left\{c_{1}, c_{2}\right\}$ be an annular pair of seiferters. When we mention the linking number $\operatorname{lk}\left(c_{1}, c_{2}\right), c_{1}$ and $c_{2}$ are oriented so as to be homologous in an annulus cobounded by $c_{1}, c_{2}$. If $c_{1} \cup c_{2}$ is not a Hopf link, then this convention determines the linking number without specifying the annulus. A Hopf link cobounds two nonisotopic annuli according as $\operatorname{lk}\left(c_{1}, c_{2}\right)=1$ or -1 . For details, see [9, Lemma 2.30 and Remark 2.31].

In [9] an annular pair $\left\{c_{1}, c_{2}\right\}$ is defined to be an ordered pair of $c_{1}$ and $c_{2}$ to specify the direction of twist along the annulus cobounded by $c_{1} \cup c_{2}$. However, since we do not perform annulus twists in this paper, annular pairs are presented as unordered pairs.

Let $K$ be a knot in $S^{3}$, and $c_{1} \cup c_{2}$ a link in $S^{3}-N(K)$. Let $c_{1}^{\prime}$ be a knot obtained from $c_{1}$ by an $m$-move using a band disjoint from $c_{2}$ and connects $c_{1}$ and a simple closed curve on $\partial N(K)$ with slope $m$. We then say that $c_{1}^{\prime} \cup c_{2}$ is obtained from $c_{1} \cup c_{2}$ by an $m$-move. The link $c_{1}^{\prime} \cup c_{2}$ is isotopic to $c_{1} \cup c_{2}$ in the surgered manifold $K(m)$ as ordered links [9, Lemma 2.25(1)]. If $\left\{c_{1}, c_{2}\right\}$ is a pair of seiferters for a Seifert surgery $(K, m)$ and $c_{1}^{\prime}$ is trivial in $S^{3}$, then $\left\{c_{1}^{\prime}, c_{2}\right\}$ is also a pair of seiferters for $(K, m)$ [9, Lemma 2.25(2)]. The theorem below complements [9, Theorem 6.21].

Theorem 3.1 (1) For each integer $m$, there are infinitely many hyperbolic Hopf pairs of seiferters for $(O, m)$.

(2) For any integers $m \neq 0$ and $p \geq 2$ except $(m, p)=( \pm 1,2)$, there is a hyperbolic annular pair of seiferters $\left\{c_{1}, c_{2}\right\}$ for $(O, m)$ with $\operatorname{lk}\left(c_{1}, c_{2}\right)=p$.

Proof of Theorem 3.1 (1) Assertion (1) follows from Lemma 3.2 below. 
(2) Assume that $m \neq 0, p \geq 2$, and $(m, p)=( \pm 1,2)$. Then, if $m \neq 1,\left\{c, c_{p+1, m}\right\}$ in Proposition 3.10 with $q$ replaced by $p+1$ is a hyperbolic annular pair for $(O, m)$ with $\operatorname{lk}\left(c, c_{p+1, m}\right)=p$, as desired in assertion (2). If $m \neq-1,\left\{c, c_{p-1, m}^{\prime}\right\}$ in Proposition 3.13 has the desired property.

Lemma 3.2 Let $O \cup c \cup c_{p}$ be the link in Figure 3.1, where $p$ is an odd integer with $|p| \geq 3$. Then, $\left\{c, c_{p}\right\}$ is a hyperbolic Hopf pair of seiferters for $(O, m)$ if $p \neq 2 m \pm 1$. For each $m,\left\{c, c_{p}\right\}(p \geq m, p \neq 2 m \pm 1)$ are mutually distinct, hyperbolic Hopf pairs.

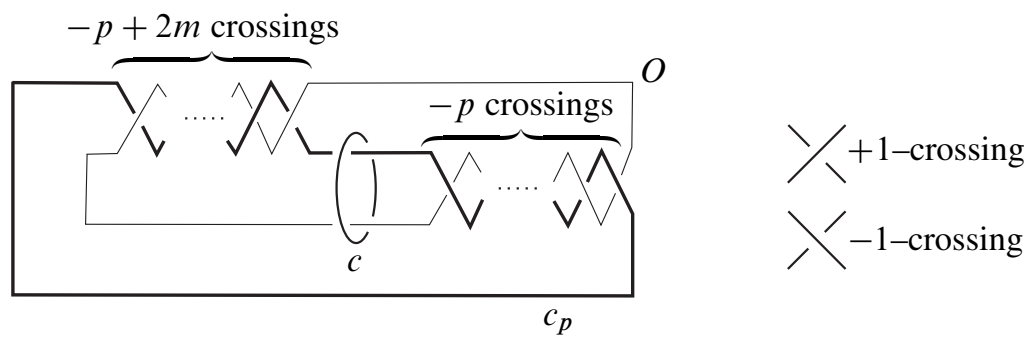

Figure 3.1

Proof of Lemma 3.2 Consider the link consisting of a torus knot $T_{p, 2}(|p| \geq 3)$ and its basic seiferters $s_{2}, s_{p}$. Regard $s_{2}$ as the trivial knot $O$, and set $c=s_{p}$. See the first figure of Figure 3.2. There is a Seifert fibration of $S^{3}-\operatorname{int} N(O)$ in which $T_{p, 2}$ is a regular fiber and $c$ is the exceptional fiber of index $|p|$. Let $c_{p}$ be the trivial knot obtained from $T_{p, 2}$ in $S^{3}-N(O)$ by the $m$-move in Figure 3.2. Since $c \cup T_{p, 2}$ is isotopic in $O(m)$ to $c \cup c_{p}, c \cup c_{p}$ is also the union of fibers in a Seifert fibration of $O(m)$. It follows that $\left\{c, c_{p}\right\}$ in the second figure of Figure 3.2 is a pair of seiferters for $(O, m)$. After isotopy, the link $O \cup c \cup c_{p}$ in the last figure of Figure 3.2 is the same link as $O \cup c \cup c_{p}$ in Figure 3.1. Hence, $\left\{c, c_{p}\right\}$ in Figure 3.1 is a Hopf pair of seiferters for $(O, m)$.

Suppose $p \neq 2 m \pm 1$. Let us show that $\left\{c, c_{p}\right\}$ is a hyperbolic Hopf pair, ie $O \cup c \cup c_{p}$ is a hyperbolic link. Assume for a contradiction that $X=S^{3}-\operatorname{int} N\left(O \cup c \cup c_{p}\right)$ is Seifert fibered. Then, the exterior of $O \cup c_{p}$, which is obtained from $X$ by Dehn filling along $\partial N(c)$, is a nondegenerate Seifert fiber space or a reducible manifold. On the other hand, since $O \cup c_{p}(p \neq 2 m \pm 1)$ is a 2-bridge link and not a torus link, it is a hyperbolic link. (For details refer to the proof of [9, Theorem 6.21].) This is a contradiction, so that $X$ is not Seifert fibered. Figure 3.3 shows that $X$ is homeomorphic to the exterior of the Montesinos link $L=M\left(1 /(2 m-p-1), \frac{1}{2}, \frac{1}{2}\right)$. The proof of Oertel [23, Corollary 5] shows that $X$ is hyperbolic if $X$ is not Seifert fibered, and $L$ is not equivalent to the 

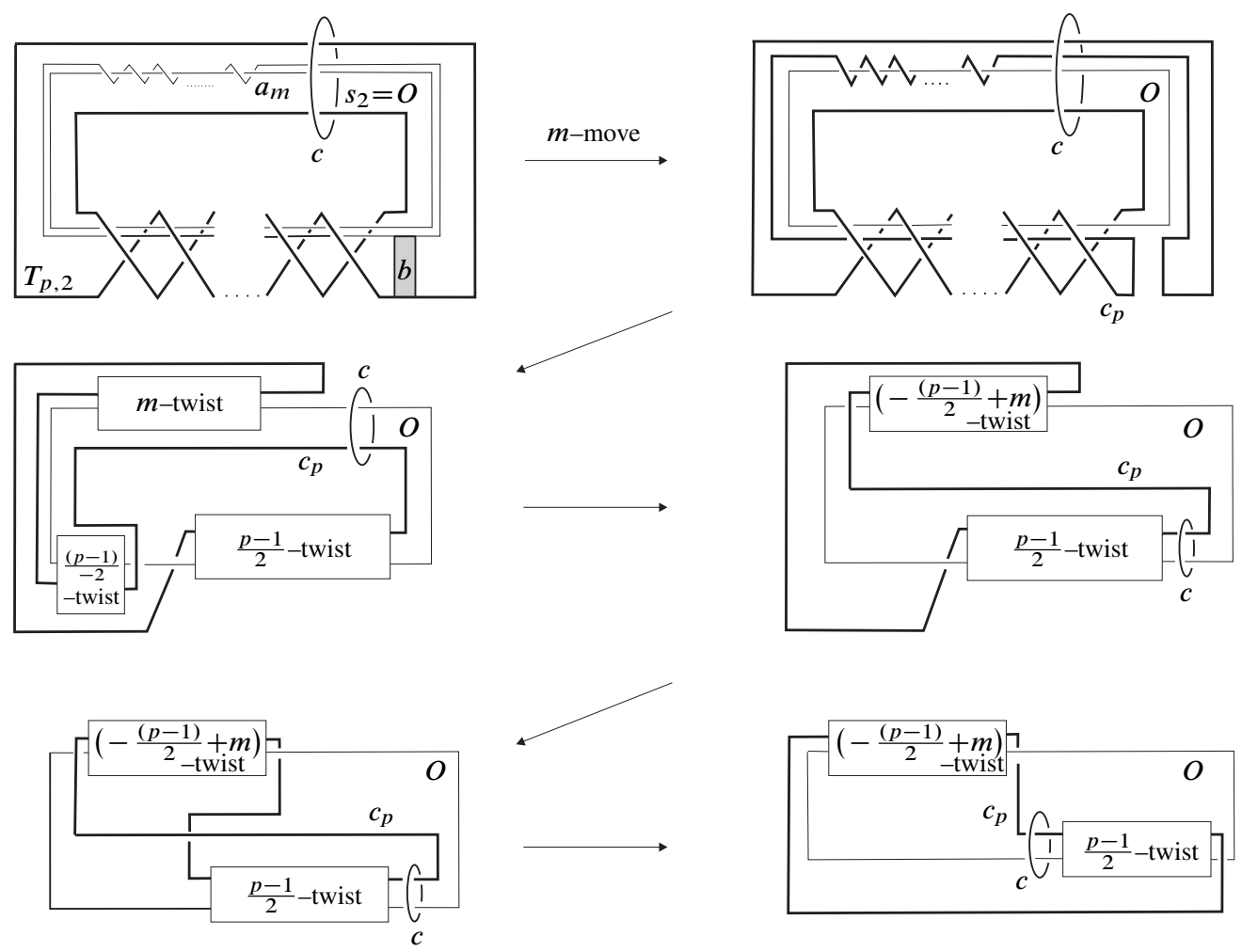

Figure 3.2

Montesinos links $M\left(\frac{1}{2}, \frac{1}{2}, \frac{-1}{2}, \frac{-1}{2}\right), M\left(\frac{2}{3}, \frac{-1}{3}, \frac{-1}{3}\right), M\left(\frac{1}{2}, \frac{-1}{4}, \frac{-1}{4}\right), M\left(\frac{1}{2}, \frac{-1}{3}, \frac{-1}{6}\right)$, or the mirror images of these links. The 2-fold branched cover of $S^{3}$ along $L$ is a prism manifold, which has a finite fundamental group. However, the 2 -fold branched covers along the four Montesinos links above have infinite fundamental groups. Therefore, $X$ is hyperbolic.

We note that $\left\{|\operatorname{lk}(c, O)|,\left|\operatorname{lk}\left(c_{p}, O\right)\right|\right\}=\{1,|m-p|\}$. Hence, if $O \cup c \cup c_{p}$ is isotopic to $O \cup c \cup c_{q}(p, q \geq m)$ in $S^{3}$ with $O$ sent to $O$, then $p=q$. It follows that for each $m$ the pairs of seiferters $\left\{c, c_{p}\right\}$ where $p \geq m$ are mutually distinct.

Remark 3.3 Corollary 5 in [23] states that a Montesinos link is hyperbolic if it is not a torus link, and not equivalent to the four Montesinos links listed above or their mirror images. However, in the proof the author assumes that links whose exteriors are Seifert fibered are torus links, which is not true. We thus obtain the corrected Corollary 5 in [23] by replacing the word "torus link" with "link whose exterior is Seifert fibered". 

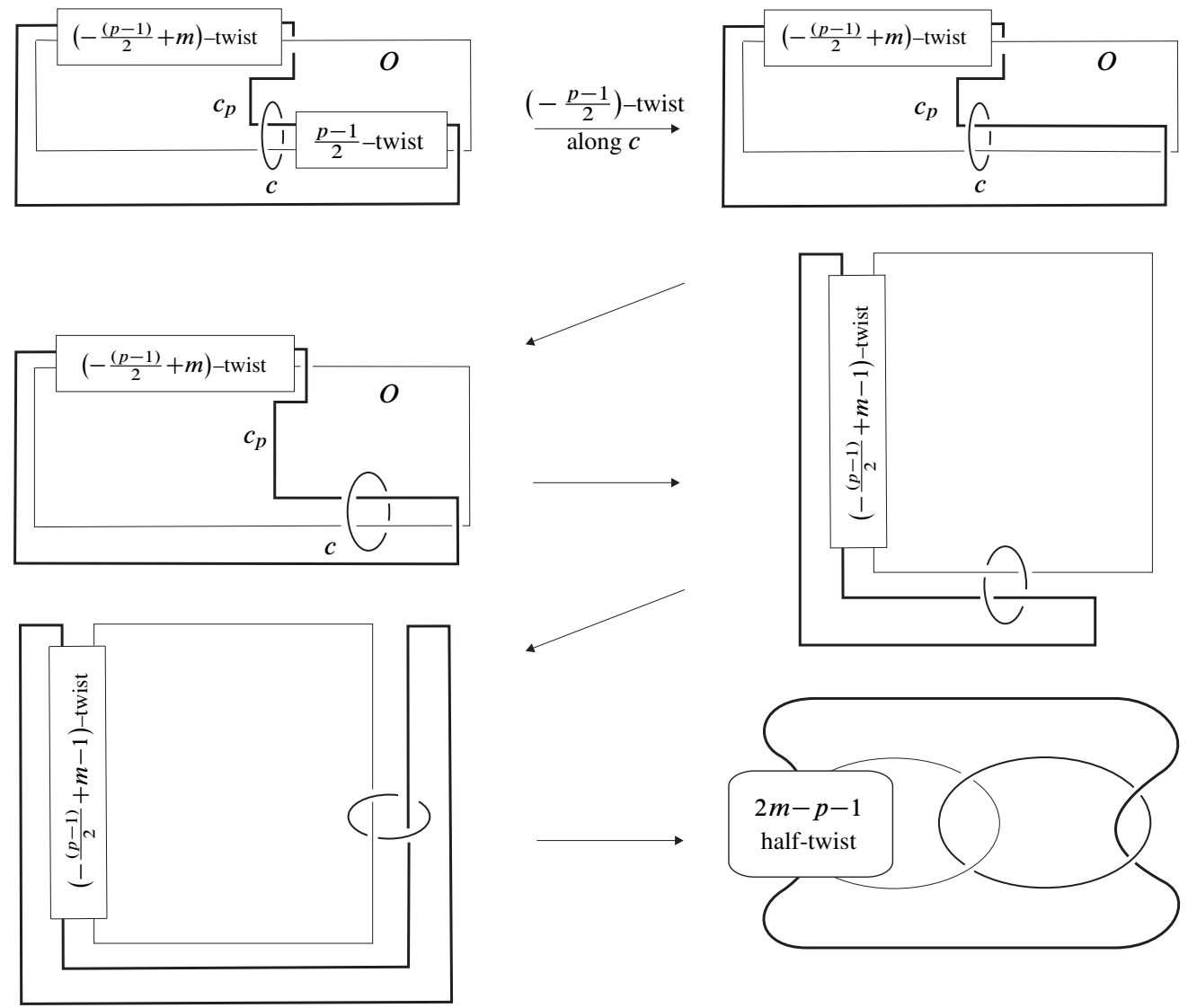

Figure 3.3: Continued from Figure 3.2.

The Hopf pair of seiferters $\left\{c, c_{p}\right\}$ satisfies $\left|\operatorname{lk}\left(c, c_{p}\right)\right|=1$. Now for a given integer $p>1$, let us find an annular pair of seiferters $\left\{c_{1}, c_{2}\right\}$ with $\operatorname{lk}\left(c_{1}, c_{2}\right)=p$ as claimed in Theorem 3.1(2). We will give such examples in Propositions 3.10 and 3.13. To prove the hyperbolicity of these examples, we prepare some general results.

Proposition 3.4 Let $l_{1} \cup \cdots \cup l_{n}$ be an $n$-component link in a solid torus $V$. Suppose that there is a meridional disk $D$ for $V$ satisfying (1), (2) below.

(1) The winding number of $l_{i}$ in $V$ equals $\left|D \cap l_{i}\right|$ for any $i$.

(2) $V-\operatorname{int} N\left(D \cup\left(\bigcup_{i=1}^{n} l_{i}\right)\right)$ is homeomorphic to a handlebody.

Then, if $V-$ int $N\left(\bigcup_{i=1}^{n} l_{i}\right)$ contains an essential torus, it bounds a solid torus in $V$. 
Proof of Proposition 3.4 We identify $V$ split along $D$ with $D^{2} \times I$, where $I=[0,1]$, and $D^{2} \times\{0\}$ and $D^{2} \times\{1\}$ are identified with $D$ in $V$. Let $a_{1}, \ldots, a_{m}$ be the arcs in $D^{2} \times I$ obtained by cutting $\bigcup_{i=1}^{n} l_{i}$ by $D$; each $a_{i}$ connects $D^{2} \times\{0\}$ and $D^{2} \times\{1\}$ by condition (1).

Assume that $V-\operatorname{int} N\left(\bigcup_{i=1}^{n} l_{i}\right)$ contains an essential torus $T$. Isotope $T$ in $V-$ int $N\left(\bigcup_{i=1}^{n} l_{i}\right)$ so as to minimize the number of components $|D \cap T|$. Note that condition (2) implies $D \cap T \neq \varnothing$. Then $D$ splits $T$ into essential annuli $A_{1}, A_{2}, \ldots, A_{k}$ properly embedded in $D^{2} \times I-\bigcup_{i=1}^{m} a_{i}$ such that a component of $\partial A_{i}$ and a component of $\partial A_{i+1}$ are identified in $V$, where $1 \leq i \leq k$, and if $i=k$ we regard $i+1=k+1$ as 1 .

Claim 3.5 Each annulus $A_{i}$ connects $D^{2} \times\{0\}$ and $D^{2} \times\{1\}$.

Proof of Claim 3.5 Assume for a contradiction that some $A_{i_{0}}$ satisfies $\partial A_{i_{0}} \subset$ $D^{2} \times\{\alpha\}$, where $\alpha=0$ or 1 . Let $B_{1}, B_{2}$ be the disks in $D^{2} \times\{\alpha\}$ bounded by the components of $\partial A_{i_{0}}$. If $B_{1} \cap B_{2}=\varnothing$, then $B_{1}$ intersects some arc $a_{j_{0}}$ and $B_{1} \cup A_{i_{0}} \cup B_{2}$ bounds a 3 -ball in $D^{2} \times I$. This implies $\partial a_{j_{0}} \subset D^{2} \times\{\alpha\}$, a contradiction to condition (1) in Proposition 3.4. It follows that $B_{1} \subset$ int $B_{2}$ or $B_{2} \subset$ int $B_{1}$. Without loss of generality, we assume that the former holds. Let $M$ be the 3-submanifold in $D^{2} \times I$ bounded by the torus $A_{i_{0}} \cup\left(B_{2}-\right.$ int $\left.B_{1}\right)$. Condition (1) then implies that $M \cap a_{i}=\varnothing$ for any $i$.

Case $1 M$ is boundary irreducible.

If $\partial M$ is incompressible in $X=V-$ int $N\left(\bigcup_{i=1}^{n} l_{i}\right)$ - int $M$, then after pushing $\partial M$ in $V-\operatorname{int} N\left(\bigcup_{i=1}^{n} l_{i}\right)$ off $D, \partial M$ is an essential torus in $D^{2} \times I-\operatorname{int} N\left(\bigcup_{i=1}^{m} a_{i}\right)$. This contradicts condition (2) in Proposition 3.4. Hence, an essential simple closed curve $c$ in $\partial M$ bounds a disk in $X$. On the other hand, $\partial B_{1}$ is also an essential simple closed curve in $\partial M$ bounding the disk $B_{1}$ in $V-\operatorname{int} M$. Since the rank of $\operatorname{Ker}\left(H_{1}(\partial M) \rightarrow H_{1}(V-\right.$ int $\left.M)\right)$ is less than or equal to one by the Poincaré duality, we see that $[c]=\left[\partial B_{1}\right]$ in $H_{1}(\partial M)$ and thus $\partial B_{1}$ bounds a disk in $X$. This contradicts the fact that $A_{i_{0}}$ is essential in $D^{2} \times I-\bigcup_{i=1}^{m} a_{i}$.

Case $2 M$ is boundary reducible.

It follows that $M$ is a solid torus. Since $\partial B_{1}(\subset \partial M)$ bounds the disk $B_{1}$ in $S^{3}$-int $M$, a meridian of $M$ and $\partial B_{1}$ intersect in one point. This implies that the annulus $A_{i_{0}}$ is parallel to $B_{1}-$ int $B_{2}$ in $M$, and contradicts the fact that $A_{i_{0}}$ is essential in $D^{2} \times I-\bigcup_{i=1}^{m} a_{i}$.

By Claim 3.5 the union of $A_{i}$ and the two disks in $D^{2} \times\{0,1\}$ bounded by $\partial A_{i}$ bounds a 3-ball $V_{i}$ in $D^{2} \times I$. Note that for any distinct $i, j$ we have $V_{i} \cap V_{j}=\varnothing$, 
$V_{i} \subset V_{j}-A_{j}$, or $V_{j} \subset V_{i}-A_{i}$. If $V_{1}, V_{2}, \ldots, V_{k}$ are mutually disjoint, then $\bigcup_{i=1}^{k} V_{i}$ forms a solid torus in $V$ bounded by $T$ as claimed in Proposition 3.4. So assume that $V_{i} \subset V_{j}-A_{j}$ for some $i, j$. Then by Claim 3.5, $V_{i+\varepsilon} \subset V_{j+\varepsilon}-A_{j+\varepsilon}$, where $\varepsilon= \pm 1$ and we regard $k+1,0$ as $1, k$, respectively. Repeating this argument, we see that for any $V_{i}$ there exists $V_{j}$ such that $V_{i} \subset V_{j}-A_{j}$. This does not occur for a finite number of 3-balls $V_{1}, V_{2}, \ldots, V_{k}$. This completes the proof of Proposition 3.4.

The following proposition will be useful.

Proposition 3.6 Let $l_{1} \cup l_{2}$ be a 2-component link in a solid torus $V$ such that $l_{1}$ is a $(p, q)$ cable of $V$ where $q \geq 2, l_{2}$ is a core of $V$ and $l_{1} \cup l_{2}$ satisfies conditions (1), (2) in Proposition 3.4. Then, $l_{1} \cup l_{2}$ is a hyperbolic link in $V$ if we cannot isotope $l_{2}$ in $V$ - int $N\left(l_{1}\right)$ so as to be disjoint from a cabling annulus for $N\left(l_{1}\right) \subset V$.

Proof of Proposition 3.6 First we remark that since $l_{i}(i=1,2)$ wraps $V$ geometrically at least once, $V-\operatorname{int} N\left(l_{1} \cup l_{2}\right)$ is irreducible.

Assume for a contradiction that $V-\operatorname{int} N\left(l_{1} \cup l_{2}\right)$ contains an essential torus $T$.

Claim 3.7 $T$ is parallel to $\partial V$ and separates $l_{1}$ and $l_{2}$, and $l_{2}$ lies between $T$ and $\partial V$.

Proof of Claim 3.7 Since $T$ is not essential in $V-$ int $N\left(l_{1}\right)$, there are three cases: (1) $T$ is compressible in $V-$ int $N\left(l_{1}\right)$, (2) $T$ is parallel to $\partial N\left(l_{1}\right)$ in $V-$ int $N\left(l_{1}\right)$ and (3) $T$ is parallel to $\partial V$ in $V-$ int $N\left(l_{1}\right)$. Case (3) implies Claim 3.7. So we derive a contradiction in cases (1), (2).

Let $V^{\prime}$ be the solid torus in $V$ bounded by $T$ (Proposition 3.4); $V^{\prime}$ contains at least one of $l_{1}$ and $l_{2}$. Since each $l_{i}$ is not contained in a 3 -ball in $V, V^{\prime}$ is not contained in a 3 -ball in $V$, either. It follows that $T=\partial V^{\prime}$ is incompressible in $V$-int $V^{\prime}$. Now assume case (1) occurs. Then $l_{2} \subset V^{\prime}$, and $T$ separates $l_{1}$ and $l_{2}$. Since $V-\operatorname{int} N\left(l_{2}\right) \cong T^{2} \times I, T$ is parallel to $\partial N\left(l_{2}\right)$ in $V-\operatorname{int} N\left(l_{1} \cup l_{2}\right)$. This contradicts the fact that $T$ is essential. Assume case (2) occurs. Since $T$ is essential in $V$-int $N\left(l_{1} \cup l_{2}\right)$, we have $l_{2} \subset V^{\prime}$. Then the winding number of $l_{2}$ in $V$ is a multiple of $q(\geq 2)$. This contradicts the fact that $l_{2}$ is a core of $V$.

Claim 3.8 For any cabling annulus $A$ for $N\left(l_{1}\right)$ in $V$, we can isotope $l_{2}$ in $V-$ int $N\left(l_{1}\right)$ so as to be disjoint from $A$.

Proof of Claim 3.8 Let $W$ be the submanifold of $V$ - int $N\left(l_{1}\right)$ cobounded by $\partial V$ and $T$. Identify $W$ and $\partial V \times I$ so that $\partial V$ and $T$ correspond to $\partial V \times\{0\}$ and $\partial V \times\{1\}$ respectively, and let $\pi: W=\partial V \times I \rightarrow I$ be the natural projection. Since $A^{\prime}=A \cap W$ 
is a compact submanifold of $W, \pi\left(A^{\prime}\right)$ is a compact and thus closed subset of $I$. It follows that $\inf \pi\left(A^{\prime}\right) \in \pi\left(A^{\prime}\right)$ and $0<\inf \pi\left(A^{\prime}\right)$, since $A \subset \operatorname{int} V$. Now isotope $l_{2} \cup T$ in $W$ so that $T$ becomes $\partial V \times\left\{\frac{1}{2} \inf \pi\left(A^{\prime}\right)\right\}$. After this isotopy $l_{2}$ becomes disjoint from $A$.

Claim 3.8 contradicts the assumption in Proposition 3.6. Hence, $V-\operatorname{int} N\left(l_{1} \cup l_{2}\right)$ contains no essential torus.

Claim 3.9 $X=V-\operatorname{int} N\left(l_{1} \cup l_{2}\right)$ contains no essential annulus.

Proof of Claim 3.9 Assume for a contradiction that $X$ contains an essential annulus. Since $X$ contains no essential torus, and is irreducible and boundary irreducible, this assumption implies that $X$ is a Seifert fiber space. Then $X$ contains an essential annulus $A$ connecting $\partial N\left(l_{1}\right)$ and $\partial V$; note that $A$ is also an essential annulus in the cable space $V-\operatorname{int} N\left(l_{1}\right)$. Take a regular neighborhood $N(\partial V \cup A)$ in $X$. Then the closure of $\partial N(\partial V \cup A)-\partial X$ is a cabling annulus for $N\left(l_{1}\right)$ in $V$. Since the cabling annulus is disjoint from $l_{2}$, this fact contradicts the assumption in Proposition 3.6.

The proof of Proposition 3.6 is thus completed.

Proposition 3.10 Let $c \cup c_{q, m}$ be the link obtained from $c \cup T_{1, q}$ in $S^{3}-N(O)$ by an $m$-move using the band $b$ in Figure 3.4(1) and an isotopy. Assume that $q \geq 3, m \neq 0,1$ and $(m, q) \neq(-1,3)$. Then, $\left\{c, c_{q, m}\right\}$ is a hyperbolic annular pair of seiferters for $(O, m)$ with $\operatorname{lk}\left(c, c_{q, m}\right)=q-1$.

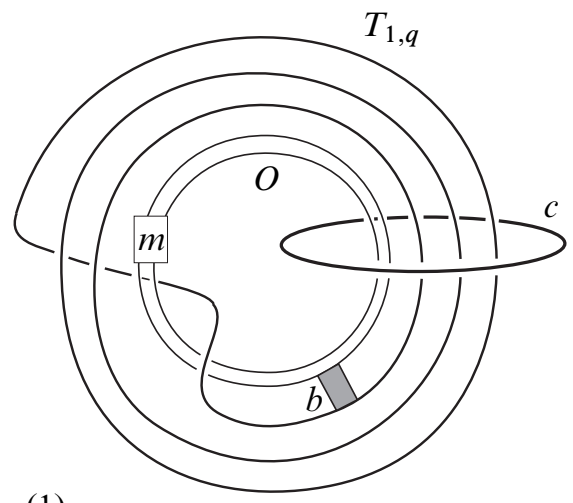

(1)

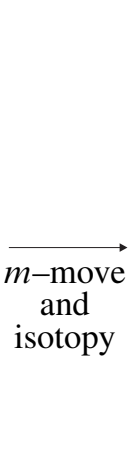

(2)

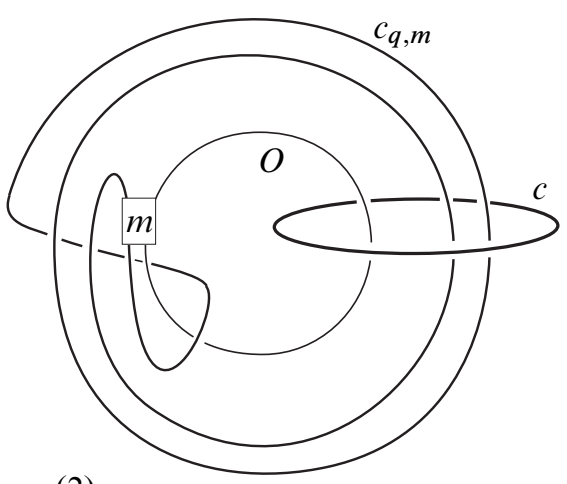

Figure 3.4: Annular pair of seiferters $\left\{c, c_{q, m}\right\} ; q=3$ 

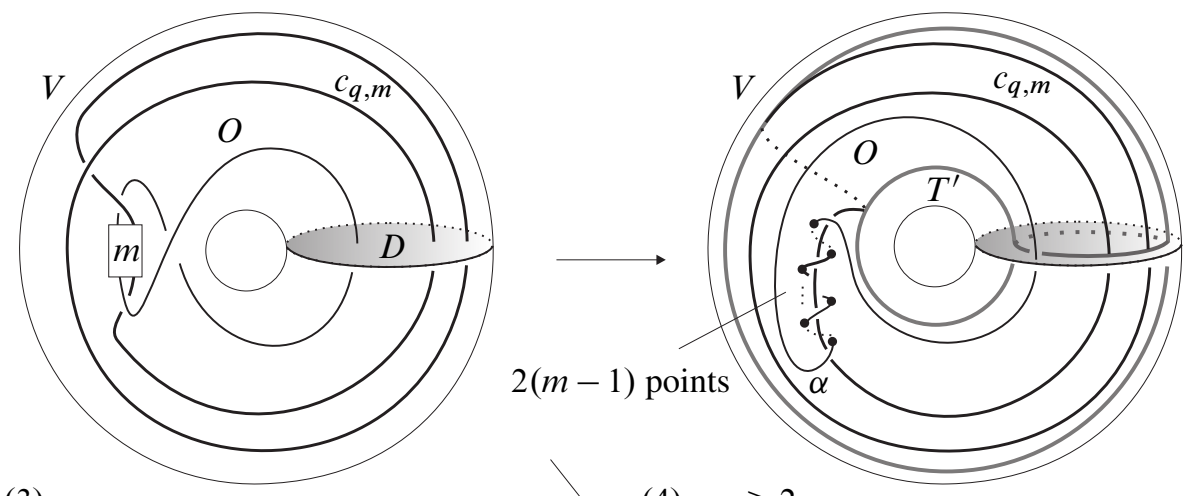

(3)

(4) $m \geq 2$

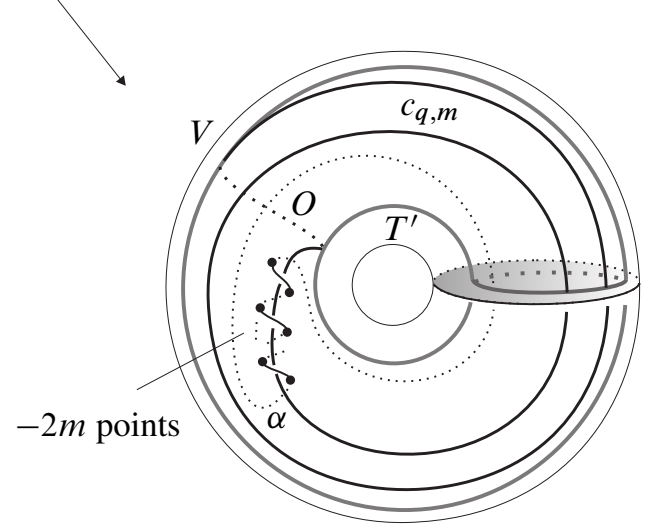

(5) $m \leq-1$

Figure 3.5: In (4), (5), the intersection points between $O$ and $T^{\prime}$ are indicated by "dots".

Proof of Proposition 3.10 Since $S^{3}$-int $N(O)$ admits a Seifert fibration in which $c$ and $T_{1, q}$ in Figure 3.4(1) are fibers, $\left\{c, T_{1, q}\right\}$ is an annular pair of seiferters for $(O, m)$. After the $m$-move in Figure 3.4, $c_{q, m}$ and $c$ in Figure 3.4(2) remain fibers in $O(m)$. Note that $c_{q, m}$ is the torus knot $T_{1, q-1}$, a trivial knot in $S^{3}$, and $c \cup c_{q, m}$ bounds an annulus. It follows that $\left\{c, c_{q, m}\right\}$ is an annular pair of seiferters for $(O, m)$. Note that $\operatorname{lk}\left(c, c_{q, m}\right)=q-1$. It remains to show that $O \cup c \cup c_{q, m}$ is a hyperbolic link.

Let $V$ be the solid torus $S^{3}-\operatorname{int} N(c)$ containing $O \cup c_{q, m}$. Then $O$ is a core of $V$ and $c_{q, m}$ is a $(1, q-1)$ cable of $V$. The meridional disk $D$ for $V$ described in Figure 3.5(3) intersects $O$ in one point and $c_{q, m}$ in $q-1$ points. Note also that $V-\operatorname{int} N\left(D \cup O \cup c_{q, m}\right)$ is homeomorphic to a handlebody. The link $O \cup c_{q, m}$ in $V$ thus satisfies conditions (1), (2) with $n=2$ in Proposition 3.4. Then by Proposition 3.6, 
in order to show that $O \cup c_{q, m}$ is hyperbolic in $V$, it is sufficient to show that $O$ cannot be isotoped in $V$-int $N\left(c_{q, m}\right)$ off a cabling annulus for $N\left(c_{q, m}\right) \subset V$.

Now let $T^{\prime}$ be the torus in $V$ containing $c_{q, m}$ as described in Figure 3.5(4), (5); then $A=T^{\prime}-\operatorname{int} N\left(c_{q, m}\right)$ is a cabling annulus for $N\left(c_{q, m}\right) \subset V$. We note that $T^{\prime}$ intersects $O$ in $2(m-1)$ points if $m \geq 2$, and in $-2 m$ points if $m \leq-1$. We denote by $\alpha$ the closure of the component of $O-T^{\prime}$ intersecting $D$. Let $V^{\prime}$ be the solid torus in $V$ bounded by $T^{\prime}$. Concerning the arc components of $O \cap V^{\prime}$ and $O \cap\left(V-\right.$ int $\left.V^{\prime}\right)$, we can check the following.

Claim 3.11 (1) In $V$-int $V^{\prime}$ (respectively $V^{\prime}$ ), the arc $\alpha$ in Figure 3.5(4) (respectively (5)) is isotopic with $\partial \alpha$ fixed to an arc in $T^{\prime}$ intersecting $c_{q, m}$ algebraically twice.

(2) In $V-\operatorname{int} V^{\prime}$ (respectively $\left.V^{\prime}\right)$, each component $\beta$ of $O \cap\left(V-\operatorname{int} V^{\prime}\right)$ (respectively $O \cap V^{\prime}$ ) other than $\alpha$ is isotopic with $\partial \beta$ fixed to an arc in $T^{\prime}$ intersecting $c_{q, m}$ once.

Using Claim 3.11, we show that there is no isotopy of $O$ in $V-\operatorname{int} N\left(c_{q, m}\right)$ which makes the intersection between $O$ and the cabling annulus $A$ empty. Assume for a contradiction that there is an isotopy $f: S^{1} \times I \rightarrow V-\operatorname{int} N\left(c_{q, m}\right)$ such that $f\left(S^{1} \times\{0\}\right)=O$ and $f\left(S^{1} \times\{1\}\right) \cap A=\varnothing$. We may assume that $f$ is transverse to $A$; then $f^{-1}(A)$ is a 1 -submanifold properly embedded in $S^{1} \times I$. Since $f\left(S^{1} \times\{1\}\right) \cap A=\varnothing$, we see $f^{-1}(A) \cap\left(S^{1} \times\{1\}\right)=\varnothing$, so that each arc component of $f^{-1}(A)$ has its end points in $S^{1} \times\{0\}$. If $f^{-1}(A)$ has a circle component bounding a disk in $S^{1} \times I$, then by the loop theorem and the incompressibility of $A$ in $V$-int $N\left(c_{q, m}\right) f$ restricted on the innermost circle is null-homotopic in $A$. Hence we can modify $f$ so that the innermost circle is eliminated. Thus by re-choosing $f$ we may assume $f^{-1}(A)$ does not contain null-homotopic circles in $S^{1} \times I$. For two arc components $a_{1}, a_{2}$ of $f^{-1}(A)$, we say that $a_{1}$ is closer to $S^{1} \times\{0\}$ than $a_{2}$ if the disk cobounded by $a_{2}$ and an arc in $S^{1} \times\{0\}$ contains $a_{1}$. Let $c_{1}$ be an arc component of $f^{-1}(A)$ closest to $S^{1} \times\{0\}$, and $c_{2}$ the arc in $S^{1} \times\{0\}$ such that $c_{1} \cup c_{2}$ cobounds a disk in $S^{1} \times I$. Note that $f\left(c_{2}\right)$ is the closure of a component of $O-A$, and $f\left(c_{1}\right)$ is an immersed arc in $A$ with $\partial f\left(c_{1}\right)=\partial f\left(c_{2}\right)$.

Claim 3.12 It holds that $q=3, m \leq-1$ and $f\left(c_{2}\right)$ is the arc $\alpha\left(\subset V^{\prime}\right)$ in Figure 3.5(5).

Proof of Claim 3.12 Set $X=V^{\prime}$ if $f\left(c_{2}\right) \subset V^{\prime}$, and $X=V-$ int $V^{\prime}$ if $f\left(c_{2}\right) \subset V-$ int $V^{\prime}$. Then $f\left(c_{1}\right)(\subset A)$ is homotopic in $X$ to the component $f\left(c_{2}\right)$ of $O \cap X$ with its end points fixed. Combining this homotopy and the isotopies in Claim 3.11, we see that $f\left(c_{1}\right)$ is homotopic in $X$ with its end points fixed to an $\operatorname{arc} \gamma$ in $T^{\prime}$ intersecting $c_{q, m}$ 
once (if $f\left(c_{2}\right)$ is an arc $\beta$ in Claim 3.11(2)) or algebraically twice (if $f\left(c_{2}\right)$ is the arc $\alpha$ in Claim 3.11(1)). Hence, the closed curve $f\left(c_{1}\right) \cup \gamma$ in $T^{\prime}$ intersecting $c_{q, m}$ once or algebraically twice is null-homotopic in $X$. Since $V-$ int $V^{\prime} \cong T^{2} \times I, f\left(c_{1}\right) \cup \gamma$, which is not null-homotopic in $T^{\prime}$, is not null-homotopic in $V$-int $V^{\prime}$. It follows that $X=V^{\prime}$ and thus $f\left(c_{2}\right) \subset V^{\prime}$. Since $c_{q, m}$ is the $(1, q-1)$ cable of $V^{\prime}$ where $q \geq 3$, a meridian of $V^{\prime}$ intersects $c_{q, m}$ algebraically $q-1$ times. It follows that $q=3$ and $\gamma$ intersects $c_{q, m}$ algebraically twice. Furthermore, we see that $f\left(c_{2}\right)$ is the arc $\alpha$ in Figure 3.5(5) and so $m \leq-1$.

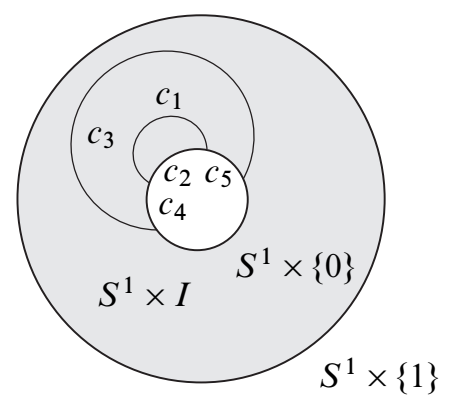

Figure 3.6

By Claim $3.12 c_{1}$ is the only arc component of $f^{-1}(A)$ closest to $S^{1} \times\{0\}$. Hence all arc components of $f^{-1}(A)$ are parallel to $c_{1}$ in $S^{1} \times I$. The assumption $(m, q) \neq$ $(-1,3)$ in Proposition 3.10 together with Claim 3.12 implies $m \leq-2$, so that $A$ intersects $O$ in $-2 m(\geq 4)$ points and hence $f^{-1}(A)$ has at least two arc components. Let $c_{3}$ be the second closest arc component of $f^{-1}(A)$ to $S^{1} \times\{0\}$, and $c_{4}, c_{5}$ the subarcs of $S^{1} \times\{0\}$ connecting $\partial c_{1}$ and $\partial c_{3}$; see Figure 3.6. Note that $f\left(c_{4}\right)$ and $f\left(c_{5}\right)$ are the components of $O \cap\left(V-\right.$ int $\left.V^{\prime}\right)$ adjacent to $f\left(c_{2}\right)=\alpha$. Now we give the $\operatorname{arcs} c_{4}$ and $c_{5}$ the orientations induced from an orientation of $S^{1} \times\{0\}$. Then, Figure 3.5(5) shows that $f\left(c_{4}\right)$ and $f\left(c_{5}\right)$ are isotopic to arcs in $T^{\prime}$ whose algebraic intersection numbers with $c_{q, m}$ are both one under an adequate orientation of $c_{q, m}$. This implies that the closed curve $f(c)$ where $c=c_{1} \cup c_{5} \cup c_{3} \cup c_{4}$ is homotopic in $V$-int $V^{\prime}$ to a closed curve in $T^{\prime}$ intersecting $c_{q, m}$ algebraically twice. Then, $f(c)$ is not null-homotopic in $V$-int $V^{\prime} \cong T^{2} \times I$. On the other hand, since $c$ bounds a disk in $S^{1} \times I$ whose image under $f$ is contained in $V-$ int $V^{\prime}, f(c)$ is null-homotopic in $V-$ int $V^{\prime}$. This is a contradiction.

Proposition 3.13 Let $c \cup c_{q, m}^{\prime}$ be the link obtained from $c \cup T_{1, q}$ in $S^{3}-N(O)$ by an $m$-move using the band $b^{\prime}$ in Figure 3.7(1) and an isotopy. Assume that $q \geq 1$, $m \neq-1,0$ and $(m, q) \neq(1,1)$. Then $\left\{c, c_{q, m}^{\prime}\right\}$ is a hyperbolic annular pair of seiferters for $(O, m)$ with $\operatorname{lk}\left(c, c_{q, m}^{\prime}\right)=q+1$. 


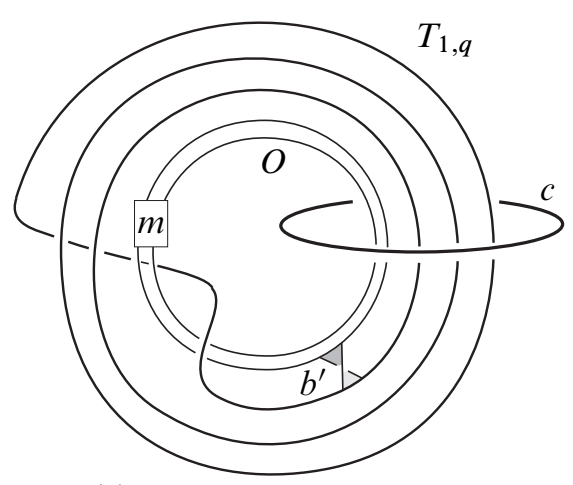

(1)

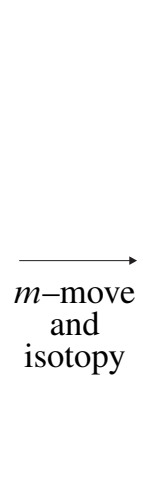

isotopy

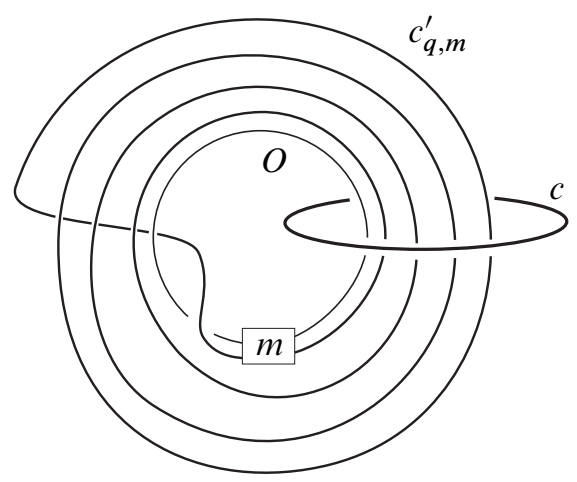

(2)

Figure 3.7: Annular pair of seiferters $\left\{c, c_{q, m}^{\prime}\right\} ; q=3$

Proof of Proposition 3.13 Apply the same argument as in the proof of Proposition 3.10 with replacement of Claims 3.11 and 3.12 by Claims 3.14 and 3.15 below.

Claim 3.14 (1) In $V^{\prime}$ (respectively $V$-int $V^{\prime}$ ), the arc $\alpha$ in Figure 3.8(4) (respectively (5)) is isotopic with $\partial \alpha$ fixed to an arc in $T^{\prime}$ intersecting $c_{q, m}^{\prime}$ algebraically twice.

(2) In $V^{\prime}$ (respectively $V-$ int $V^{\prime}$ ), each component $\beta$ of $O \cap V^{\prime}$ (respectively $O \cap\left(V-\right.$ int $\left.\left.V^{\prime}\right)\right)$ other than $\alpha$ is isotopic with $\partial \beta$ fixed to an arc in $T^{\prime}$ intersecting $c_{q, m}^{\prime}$ once.

Claim 3.15 It holds that $q=1, m \geq 1$ and $f\left(c_{2}\right)$ is the $\operatorname{arc} \alpha\left(\subset V^{\prime}\right)$ in Figure 3.8(4).

Remark 3.16 Assume that $p \geq 2, m \neq 0, \pm 1$. Then $\left\{c, c_{p+1, m}\right\}$ in Proposition 3.10 and $\left\{c, c_{p-1, m}^{\prime}\right\}$ in Proposition 3.13 are both hyperbolic annular pairs of seiferters for $(O, m)$ with $\operatorname{lk}\left(c, c_{p+1, m}\right)=\operatorname{lk}\left(c, c_{p-1, m}^{\prime}\right)=p$. Since $\left\{|\operatorname{lk}(c, O)|,\left|\operatorname{lk}\left(c_{p+1, m}, O\right)\right|\right\}=$ $\{1,|1-m|\}$ does not coincide with $\left\{|\operatorname{lk}(c, O)|,\left|\operatorname{lk}\left(c_{p-1, m}^{\prime}, O\right)\right|\right\}=\{1,|1+m|\}$, then $\left\{c, c_{p+1, m}\right\}$ and $\left\{c, c_{p-1, m}^{\prime}\right\}$ are distinct, annular pairs for $(O, m)$.

\section{Seiferters and Hopf pairs for $\left(T_{p, 2}, m\right)$}

Theorem 4.1 For nontrivial torus knots $T_{p, 2}(|p| \geq 3)$, the following hold.

(1) Each Seifert surgery $\left(T_{p, 2}, m\right)$ has a hyperbolic Hopf pair of seiferters.

(2) A Seifert surgery $\left(T_{p, 2}, m\right)$ has a hyperbolic seiferter if $m \neq 2 p \pm 1$ and $(m, p) \neq$ $(4,3),(-4,-3)$. 

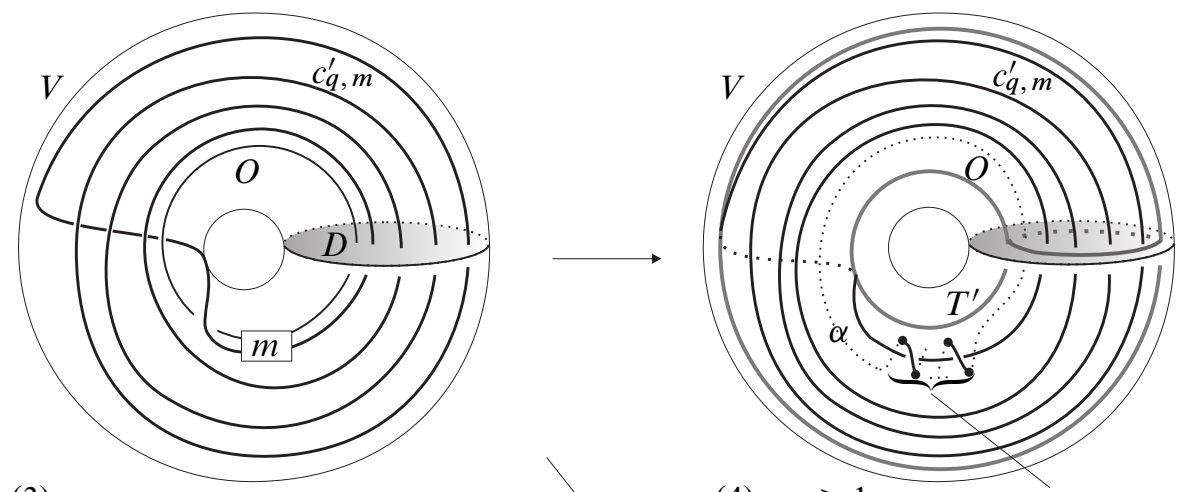

(3)

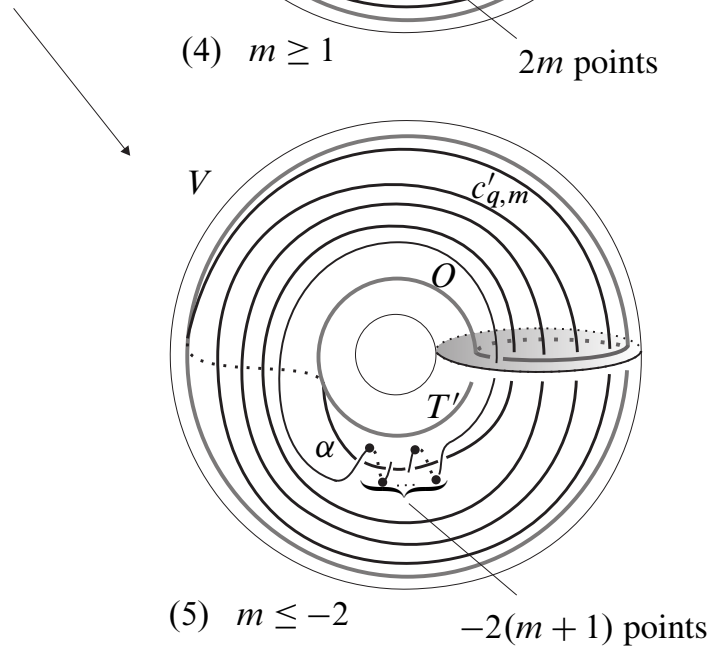

Figure 3.8: In (4), (5), the intersection points between $O$ and $T^{\prime}$ are indicated by "dots".

Proof of Theorem 4.1 Theorem 4.1(1) follows from Proposition 4.2(1) below.

Theorem 4.1(2) follows from Proposition 4.2(2) if $|p| \geq 5$ and $m \neq 2 p$. The case when $m=2 p$ follows from the fact that $\left(T_{p, q}, p q\right)$ has a hyperbolic seiferter for any nontrivial torus knot $T_{p, q}$ (Claim 5.2 and the second and third author [21, Lemma 9.1]). The remaining case is when $|p|=3$. For trefoil knots, various seiferters and annular pairs are found in [7]. For example, we see from Remark 4.6(1) that $\left(T_{3,2}, m\right)$ (respectively $\left.\left(T_{-3,2}, m\right)\right)$ has a hyperbolic seiferter if $m \neq 4$ (respectively $m \neq-4$ ). This shows Theorem 4.1(2) with $|p|=3$.

Proposition 4.2 Let $c_{m}$ be the knot obtained from the basic seiferter $s_{2}$ for $\left(T_{p, 2}, m\right)$ $(|p| \geq 3)$ by an $m$-move using the band $b$ described in Figure 4.1. Then the following hold. 
(1) For the meridional seiferter $c_{\mu}$ as in Figure 4.1, $\left\{c_{\mu}, c_{m}\right\}$ is a hyperbolic Hopf pair of seiferters for $\left(T_{p, 2}, m\right)$.

(2) The knot $c_{m}$ is a hyperbolic seiferter for $\left(T_{p, 2}, m\right)$ if $|p| \geq 5$ and $m \neq 2 p, 2 p \pm 1$.

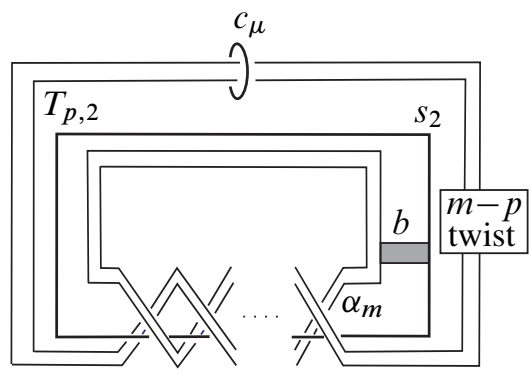

Figure 4.1: $c_{m}=s_{2} \natural_{b} \alpha_{m}$

Remark 4.3 If $m=2 p$, then $c_{m}$ in Proposition 4.2 is the same as the basic seiferter $s_{2}$ for $T_{p, 2}$. If $m=2 p \pm 1$, then $c_{m}$ is a $\left(1, \frac{p \pm 1}{2}\right)$ cable of $s_{p}$ for $T_{p, 2}$, ie $c_{m}$ is the seiferter $s_{p, \pm 1}$ for $\left(T_{p, 2}, 2 p \pm 1\right)$ defined in [9, Corollary 3.15(2)].

Proof of Proposition 4.2 In (1) we may assume that $p \geq 3$ because the corresponding result for $p \leq-3$ can be derived by taking mirror images. For the same reason we may assume $p \geq 5$ in (2).

(1) The sequence of isotopies in Figures 4.2 and 4.3 shows that $c_{m}$ is a trivial knot. Since $c_{m}$ is obtained from $s_{2}$ by an $m$-move and $T_{p, 2}$ is a nontrivial knot, $c_{m}$ is a seiferter for $\left(T_{p, 2}, m\right)$ by [9, Proposition 2.19(3)]. Furthermore, since $\left\{c_{\mu}, s_{2}\right\}$ is a pair of seiferters for $\left(T_{p, 2}, m\right)$ and the band $b$ is disjoint from $c_{\mu}$ in Figure 4.1, $\left\{c_{\mu}, c_{m}\right\}$ is a pair of seiferters [9, Lemma 2.25(2)]. The last figure of Figure 4.4 shows that $\left\{c_{\mu}, c_{m}\right\}$ is a Hopf pair of seiferters. Let us verify that an annulus cobounded by $c_{\mu}$ and $c_{m}$ intersects $T_{p, 2}$ if $m \neq p \pm 1$. This implies that $\left\{c_{\mu}, c_{m}\right\}(m \neq p \pm 1)$ is not irrelevant and thus an annular pair (Remark 1.3); in particular, $\left\{c_{\mu}, c_{0}\right\}$ is an annular pair of seiferters. Since $\left|\operatorname{lk}\left(c_{m}, T_{p, 2}\right)\right|=|m-p| \neq 1=\left|\operatorname{lk}\left(c_{\mu}, T_{p, 2}\right)\right|, c_{m}$ is not homologous to $c_{\mu}$ in $S^{3}-T_{p, 2}$. It follows that $c_{\mu}$ and $c_{m}$ does not cobound an annulus disjoint from $T_{p, 2}$, as desired.

Let us show that $\left\{c_{\mu}, c_{m}\right\}$ is a hyperbolic annular pair for any $m$. In the last figure of Figure 4.4, $(-m)$-twist along $c_{\mu}$ changes $T_{p, 2} \cup c_{m}$ to $T_{p, 2} \cup c_{0}$. Hence, there is an orientation preserving homeomorphism from $S^{3}-T_{p, 2} \cup c_{\mu} \cup c_{m}$ to $S^{3}-T_{p, 2} \cup c_{\mu} \cup c_{0}$. Thus it is sufficient to show that $S^{3}-T_{p, 2} \cup c_{\mu} \cup c_{0}$ is hyperbolic. Since $c_{\mu} \cup c_{0}$ 


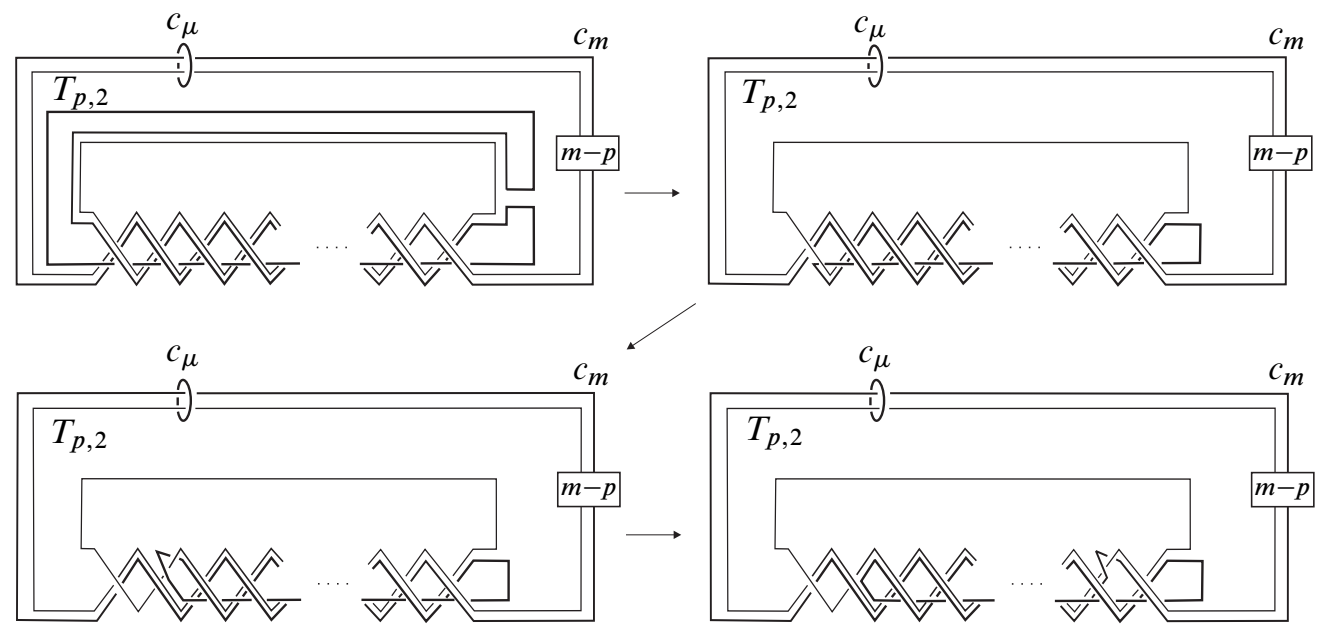

Figure 4.2: Isotopy of $T_{p, 2} \cup c_{\mu} \cup c_{m}$
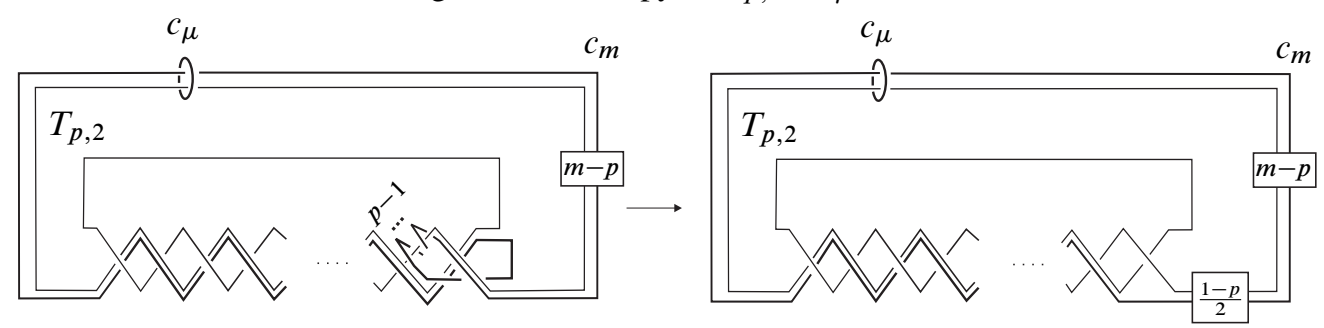

Figure 4.3: Continued from Figure 4.2

is isotopic to $c_{\mu} \cup s_{2}$ in $T_{p, 2}(0), c_{\mu}$ and $c_{0}$ are exceptional fibers of indices $2 p$ and 2, respectively in the small Seifert fiber space $T_{p, 2}(0)$ over $S^{2}(2, p, 2 p)$. Then apply [9, Theorem 3.24] to the annular pair $\left\{c_{\mu}, c_{0}\right\}$. We see that $\left\{c_{\mu}, c_{0}\right\}$ is a hyperbolic annular pair or a basic annular pair, ie a pair of basic seiferters $c_{\mu}, s_{2}, s_{p}$ as drawn in Figure 1.1. However, the latter does not occur because $\left|\operatorname{lk}\left(c_{\mu}, c_{0}\right)\right|=1$. Hence, $\left\{c_{\mu}, c_{0}\right\}$ and thus $\left\{c_{\mu}, c_{m}\right\}$ are hyperbolic annular pairs for $\left(T_{p, 2}, m\right)$.

(2) Assume that $m \neq 2 p, 2 p \pm 1$ and $p \geq 5$. As shown in (1) $c_{m}$ is isotopic to $s_{2}$ in $T_{p, 2}(m)$, and thus an exceptional fiber of index 2 in $T_{p, 2}(m)$, a Seifert fiber space over $S^{2}(2, p,|2 p-m|)$. Then, [9, Corollary 3.15] shows that $c_{m}$ is either a basic or a hyperbolic seiferter. Therefore, Claim 4.4 below implies that $c_{m}$ is a hyperbolic seiferter for $\left(T_{p, 2}, m\right)$, as claimed in Proposition 4.2(2).

Claim 4.4 The seiferter $c_{m}$ in Figure 4.1 is not a basic seiferter for $\left(T_{p, 2}, m\right)$.

Proof of Claim 4.4 We observe the following from the last figure in Figure 4.4. 

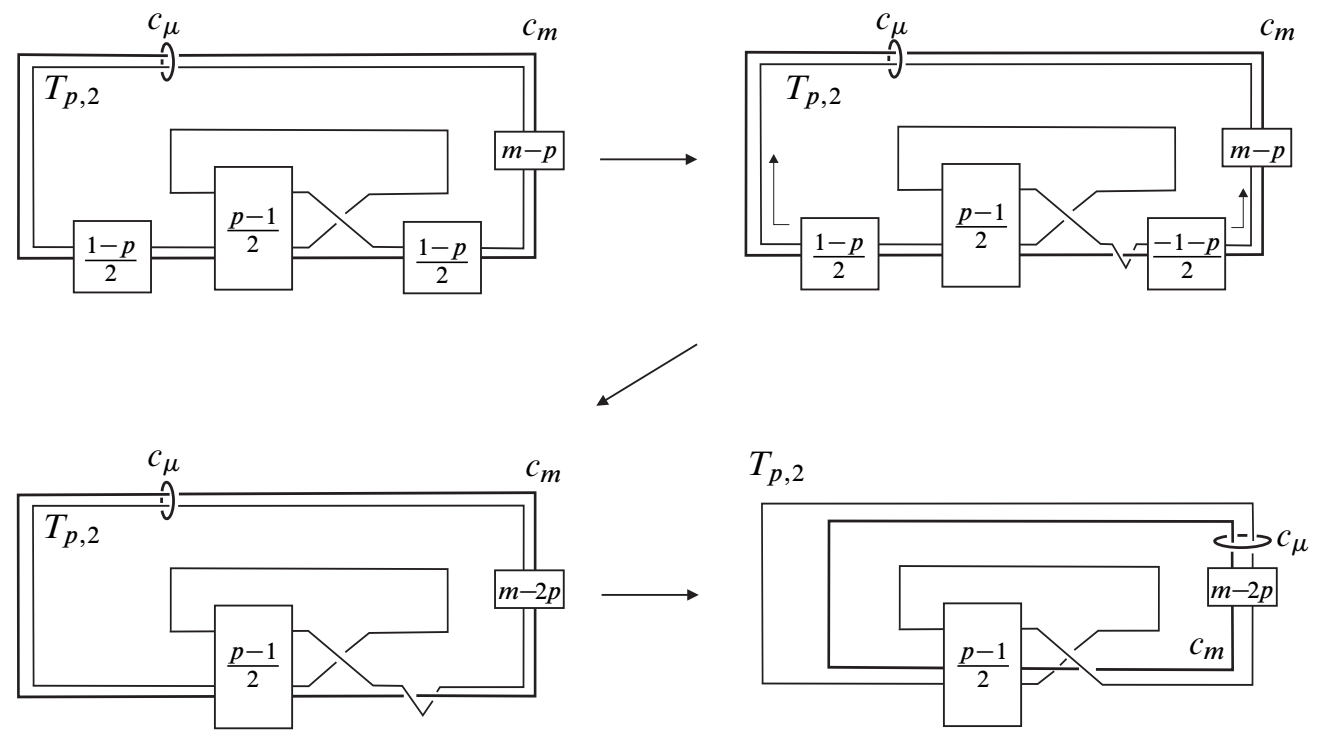

Figure 4.4: Continued from Figure 4.3

(i) The seiferter $c_{2 p}$ is the same as the basic seiferter $s_{2}$ for $T_{p, 2}$.

(ii) The link $T_{p, 2} \cup c_{m}$ is obtained from $T_{p, 2} \cup s_{2}$ after $(m-2 p)$-twist along $c_{\mu}$.

Let $M=S^{3}-$ int $N\left(T_{p, 2} \cup c_{\mu} \cup c_{2 p}\right) ; M$ is proved to be hyperbolic in the proof of Proposition 4.2(1). We see from observations (i), (ii) above that the $1 /(2 p-m)-$ Dehn filling $M(1 /(2 p-m))$ along $\partial N\left(c_{\mu}\right)$ is homeomorphic to $S^{3}-\operatorname{int} N\left(T_{p, 2} \cup c_{m}\right)$, and $M\left(\frac{1}{0}\right) \cong S^{3}-\operatorname{int} N\left(T_{p, 2} \cup s_{2}\right)$ is a Seifert fiber space. Now assume for a contradiction that $c_{m}$ is a basic seiferter for $T_{p, 2}$; then $S^{3}-\operatorname{int} N\left(T_{p, 2} \cup c_{m}\right)$ is Seifert fibered. By Gordon and $\mathrm{Wu}[13$, Corollary 1.2] we obtain $|2 p-m| \leq 3$. Since $m \neq 2 p, 2 p \pm 1$, it follows $|2 p-m|=2$ or 3 .

Assume $|2 p-m|=2$; then $\left|\operatorname{lk}\left(c_{m}, T_{p, 2}\right)\right|=m-p=p+2(>0)$ or $p-2(>0)$. If $c_{m}$ is the same as $s_{2}$, then we have $\left|\operatorname{lk}\left(c_{m}, T_{p, 2}\right)\right|=\left|\operatorname{lk}\left(s_{2}, T_{p, 2}\right)\right|=p$, a contradiction. If $c_{m}$ is the same as $s_{p}$, then we have $\left|\operatorname{lk}\left(c_{m}, T_{p, 2}\right)\right|=2$, so that $p=0$ or 4 . This is not the case because $p$ is an odd integer. If $c_{m}$ is the same as $c_{\mu}$, then since $\left|\operatorname{lk}\left(c_{\mu}, T_{p, 2}\right)\right|=1$, we obtain $p=-1,3$. This contradicts the assumption $p \geq 5$.

Assume $|2 p-m|=3$; then $\left|\operatorname{k}\left(c_{m}, T_{p, 2}\right)\right|=m-p=p+3(>0)$ or $p-3(>0)$. By comparing linking numbers as above, we can see that $c_{m}$ is distinct from $s_{2}, c_{\mu}$, and thus $c_{m}$ is the same as $s_{p}$ and $p=5$. Since $c_{m}$ is also isotopic to $s_{2}$ in $T_{p, 2}(m), T_{p, 2}(m)-$ int $N\left(s_{2}\right)$ (a Seifert fiber space over $\left.D^{2}(p,|2 p-m|)=D^{2}(5,3)\right)$ is homeomorphic to $T_{5,2}(m)-\operatorname{int} N\left(s_{5}\right)$ (a Seifert fiber space over $D^{2}(2,3)$ ). This 
homeomorphism does not preserve Seifert fibrations up to isotopy, a contradiction to [19, Theorem VI.18].

As for $T_{-3,2}$ we find various seiferters and annular pairs of seiferters in [7].

Proposition 4.5 [7] Take the knot $c^{m}$ in $S^{3}-T_{3,2}$ illustrated in Figure 4.5; then $c^{m}$ is a hyperbolic seiferter for $\left(T_{3,2}, m\right),\left(T_{3,2}, m+1\right)$ and $\left(T_{3,2}, m+2\right)$ except when $m=2,3,4,5$. In particular, $\left(T_{3,2}, m\right)$ has a hyperbolic seiferter if $m \neq 4,5$.

Remark 4.6 (1) By setting $n=2$ in Proposition 5.5, we see that $\left(T_{3,2}, 5\right)$ has a hyperbolic seiferter. This together with Proposition 4.5 shows that $\left(T_{3,2}, m\right)$ has a hyperbolic seiferter for $m \neq 4$.

(2) The seiferter $c^{m}$ in Figure 4.5 for $\left(T_{3,2}, m\right)$ is isotopic in $S^{3}-T_{3,2}$ to the seiferter $c_{m+2}$ for $\left(T_{3,2}, m+2\right)$ in Figure 4.1.

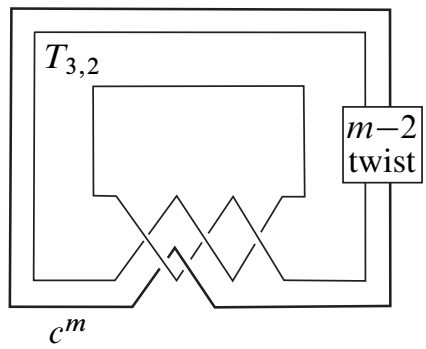

Figure 4.5: Seiferter $c^{m}\left(=c_{m+2}\right)$ for $\left(T_{3,2}, m\right)$

\section{Seiferters not originating in Seifert fibrations of torus knot spaces}

As shown in Proposition 2.2, if $m \neq p q, p q \pm 1$ and $T_{p, q}(m)$ is not a prism manifold, then any seiferter for $\left(T_{p, q}, m\right)$ is $m$-equivalent to a basic seiferter or a regular fiber of $S^{3}-N\left(T_{p, q}\right)$. On the contrary, as shown in this section, there exist seiferters for $\left(T_{p, q}, m\right)$ which cannot be obtained from basic seiferters or regular fibers by a sequence of $m$-moves. In fact, for all $T_{p, q}$ but $T_{ \pm 3,2}$ the degenerate Seifert surgery $\left(T_{p, q}, p q\right)$ has a hyperbolic seiferter not $p q$-equivalent to a basic seiferter or a regular fiber in $S^{3}-N\left(T_{p, q}\right)$; for some $T_{p, q}$ Seifert surgeries $\left(T_{p, q}, m\right)$ where $m=p q+1$ or $p q-1$ have such seiferters. Examples of the former statement will be given in Proposition 5.1, and those of the latter in Propositions 5.4, 5.5. 
Proposition 5.1 Each Seifert surgery $\left(T_{p, q}, p q\right)(|p|>q \geq 2)$ where $(p, q) \neq( \pm 3,2)$ has a hyperbolic seiferter which is not $p q$-equivalent to any basic seiferter for $T_{p, q}$ or a regular fiber of $S^{3}-N\left(T_{p, q}\right)$. Furthermore, if $|p+q|$ and $|p-q|$ are both greater than one, then $\left(T_{p, q}, p q\right)$ has at least two such hyperbolic seiferters.

Proof of Proposition 5.1 Let $c_{+}, c_{-}$be the knots in the exterior of a nontrivial torus knot $T_{p, q}$ as described in Figure 5.1. The link $T_{p, q} \cup c_{+}$is exactly the same as the link $T_{p, q} \cup c$ in [9, Figure 4.2]; see also [21, Figure 13]. Note that $\operatorname{lk}\left(c_{+}, T_{p, q}\right)=p+q$ and $\operatorname{lk}\left(c_{-}, T_{p, q}\right)=p-q$. The result on $c_{+}$in Claim 5.2 below is essentially obtained in [21, Lemma 9.1]. Since the link $T_{p, q} \cup c_{-}$is the mirror image of $T_{-p, q} \cup c_{+}$, the statement on $c_{-}$also holds.

Claim 5.2 The knots $c_{ \pm}$are seiferters for $\left(T_{p, q}, p q\right)$. Each of $c_{ \pm}$is a degenerate Seifert fiber in $T_{p, q}(p q)$ such that $T_{p, q}(p q)$ - int $N\left(c_{ \pm}\right)$is a Seifert fiber space over the disk with two exceptional fibers of indices $|p|, q$. Furthermore, if $|p+q| \neq 1$ (respectively $|p-q| \neq 1$ ), then $c_{+}$(respectively $c_{-}$) is a hyperbolic seiferter for $\left(T_{p, q}, p q\right)$; otherwise, $c_{+}$(respectively $\left.c_{-}\right)$is a meridian of $T_{p, q}$.
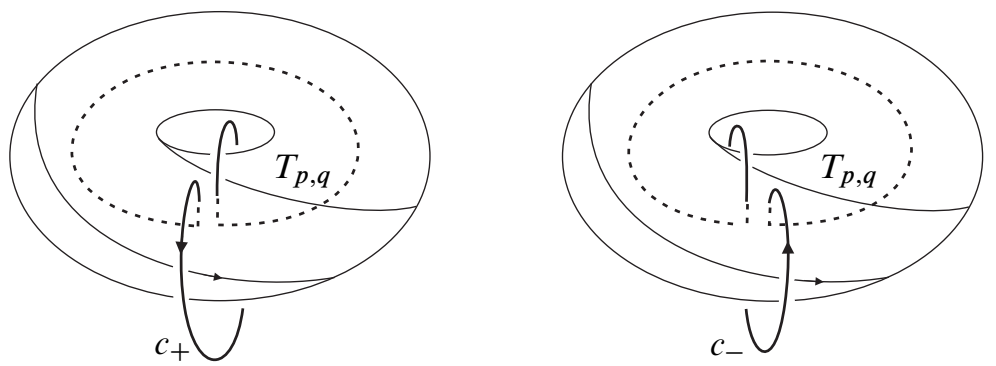

Figure 5.1: Hyperbolic seiferters $c_{+}$and $c_{-}$for $\left(T_{p, q}, p q\right)$

Since there are no $p, q(|p|>q \geq 2)$ satisfying $|p+q|=|p-q|=1$, at least one of $c_{+}, c_{-}$is a hyperbolic seiferter for $\left(T_{p, q}, p q\right)$. Set $c=c_{+}$if $|p+q| \neq 1$, and otherwise $c=c_{-}$.

Let us show that $c$ is not $p q$-equivalent to any basic seiferter if $(p, q) \neq(3, \pm 2)$. If $c$ were $p q$-equivalent to $s_{p}$ (respectively $s_{q}$ ), then the Seifert fiber space $T_{p, q}(p q)-$ int $N(c)$ would be homeomorphic to $T_{p, q}(p q)-$ int $N\left(s_{p}\right) \cong S^{1} \times D^{2} \sharp L(q, p)$ (respectively $T_{p, q}(p q)-$ int $N\left(s_{q}\right) \cong S^{1} \times D^{2} \sharp L(p, q)$ ), a contradiction to Claim 5.2. If $c$ were $p q$-equivalent to a meridional seiferter $c_{\mu}$, then [9, Proposition 2.22(1)] would show that $\operatorname{lk}\left(c, T_{p, q}\right)= \pm 1+x p q$ for some integer $x$. Since $\operatorname{lk}\left(c, T_{p, q}\right)=p \pm q$, a simple computation shows $(p, q)=( \pm 3,2)$, a contradiction to our assumption. If $c$ 
were $p q$-equivalent to a regular fiber $t$ in $S^{3}-N\left(T_{p, q}\right)$, then the Seifert fiber space $T_{p, q}(p q)-\operatorname{int} N(c)$ would be homeomorphic to $T_{p, q}(p q)-\operatorname{int} N(t) \cong S^{1} \times D^{2} \#$ $L(p, q) \sharp L(q, p)$, a contradiction.

Suppose we have that $|p+q|$ and $|p-q|$ are both greater than one. We then see that $c_{+}$and $c_{-}$are both hyperbolic seiferters for $\left(T_{p, q}, p q\right)$ with the required property. Since $\left|\operatorname{kk}\left(c_{+}, T_{p, q}\right)\right|=|p+q| \neq|p-q|=\left|\operatorname{kk}\left(c_{-}, T_{p, q}\right)\right|, c_{+}$and $c_{-}$are distinct seiferters.

Remark $5.3\left(T_{p, q}, p q\right)$ may have a hyperbolic seiferter other than $c_{+}$and $c_{-}$. For example, $\left(T_{3,5}, 15\right)$ has a hyperbolic seiferter $c$ such that $\operatorname{lk}\left(c, T_{3,5}\right)=4$. Since $4 \neq|3 \pm 5|, c$ is neither $c_{+}$nor $c_{-}$. See [9, Remark 9.20(1)].

A seiferter for $\left(T_{p, q}, p q\right)$ which is not $p q$-equivalent to any basic seiferter or a regular fiber of $S^{3}-N\left(T_{p, q}\right)$ arises because of nonuniqueness of degenerate Seifert fibrations of $T_{p, q}(p q)$. Similarly, nonuniqueness of Seifert fibrations of lens spaces make it possible for some lens surgeries to have such seiferters.

Proposition 5.4 The lens surgery $\left(T_{2 n+1, n}, n(2 n+1)-1\right)(n \geq 2)$ has a hyperbolic seiferter which is not $(n(2 n+1)-1)$-equivalent to any basic seiferter for $T_{2 n+1, n}$ or a regular fiber of $S^{3}-N\left(T_{2 n+1, n}\right)$.

Proof of Proposition 5.4 In [8, Proposition 3.7], we prove that $c$ described in Figure 5.2(1) is a seiferter for the lens surgery $\left(T_{-2 n-3, n+2},(-2 n-3)(n+2)+1\right)$. Twisting $T_{-2 n-3, n+2}$ once along the seiferter $c$, we obtain Figure 5.2(2). Figure 5.3 demonstrates that the image of $T_{-2 n-3, n+2}$ after the twisting is $T_{2 n+1, n}$; since $\operatorname{lk}\left(c, T_{-2 n-3, n+2}\right)=2 n+2$, the resulting surgery slope is $(-2 n-3)(n+2)+1+$ $(2 n+2)^{2}=n(2 n+1)-1$. Thus we obtain the lens surgery $\left(T_{2 n+1, n}, n(2 n+1)-1\right)$ for which $c$ remains a seiferter. Note that $\operatorname{lk}\left(c, T_{2 n+1, n}\right)=\operatorname{lk}\left(c, T_{-2 n-3, n+2}\right)=2 n+2$.

Let $\left(K_{p}, m_{p}\right)$ be the Seifert surgery obtained from $\left(T_{2 n+1, n}, n(2 n+1)-1\right)$ after a $p$-twist along $c$. Then $\left(K_{p}, m_{p}\right)(p \in \mathbb{Z})$ are Berge's lens surgeries on type III knots. Proposition 3.8 in [8] shows that each lens space $K_{p}\left(m_{p}\right)$ has a Seifert fibration $\mathcal{F}$ over $S^{2}$ such that $\mathcal{F}$ has two exceptional fibers and $c$ (the image of $c$ after twisting) is a regular fiber of $\mathcal{F}$. Hence, $K_{p}\left(m_{p}\right)-$ int $N(c)$ is a Seifert fiber space over the disk with two exceptional fibers.

Since $\operatorname{lk}\left(c, T_{2 n+1, n}\right)=2 n+2 \notin\{1, n, 2 n+1\}$, the seiferter $c$ is not a basic seiferter for $T_{2 n+1, n}$. Then, if $c$ were not a hyperbolic seiferter for $\left(K_{0}, m_{0}\right)=\left(T_{2 n+1, n}\right.$, $n(2 n+1)-1)$, case (2), (4), (5), (6) or (7) in [9, Corollary 3.15$]$ would occur. 


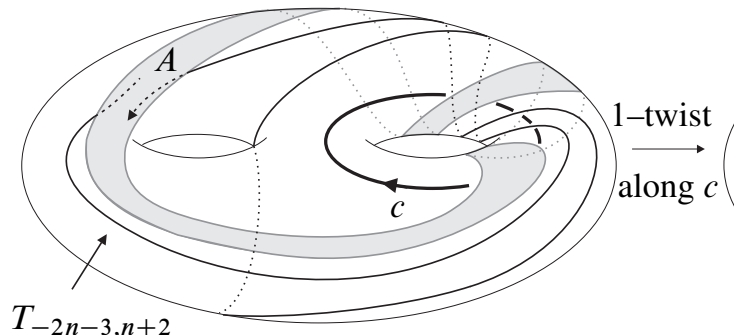

(1)

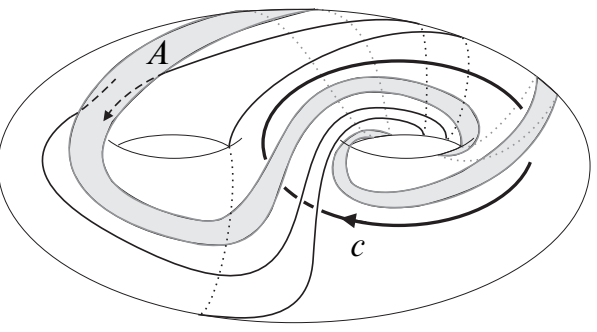

(2)

Figure 5.2: An $n$-Dehn twist is performed along the annulus $A$.

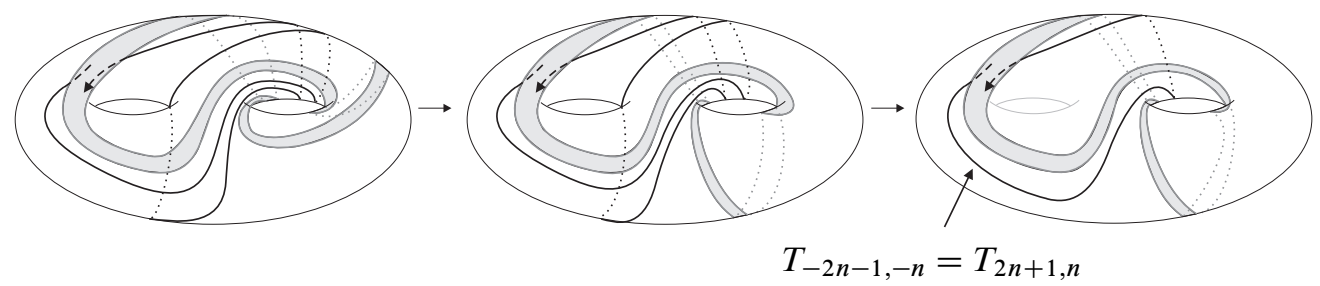

Figure 5.3

In these cases, $c$ is a $(1, x)$ cable $(|x| \geq 2)$ of an unknotted solid torus $V$ in $S^{3}, K_{0}$ is a knot in $U=S^{3}-$ int $V$, and a Seifert fibration of $K_{0}\left(m_{0}\right)$ restricts to that of $V$ with $c$ a regular fiber. Now for a knot $\mathrm{k}$ in a 3 -manifold $X\left(\subset S^{3}\right)$, let us denote by $X(k ; \gamma)$ the manifold obtained from $X$ after $\gamma$-surgery on $k$. If $|p| \geq 2$, then $V\left(c ;-\frac{1}{p}\right)$ has a Seifert fibration over the disk in which a core of the filled solid torus is an exceptional fiber of index $|p x+1|$ and a core of $V$ is another exceptional fiber of index $|x|$. In cases (2), (4), (5) and (7), $U\left(K_{0} ; m_{0}\right)$ has a Seifert fibration over the disk with at most two exceptional fibers. Hence $K_{p}\left(m_{p}\right)=U\left(K_{0} ; m_{0}\right) \cup V\left(c ;-\frac{1}{p}\right)$ is either a Seifert fiber space with more than two exceptional fibers or a lens space which has two exceptional fibers with $c$ (the image of $c$ after a $p$-twist) one of them. The former case contradicts the fact that $\left(K_{p}, m_{p}\right)$ is a lens surgery for any $p$. The latter implies $K_{p}\left(m_{p}\right)$-int $N(c)$ is a solid torus, a contradiction. The remaining case is (6) in [9, Corollary 3.15]. In this case, $K_{0}\left(m_{0}\right)-$ int $N(c)$ is a Seifert fiber space over the Möbius band with one exceptional fiber, a contradiction. It follows that $c$ is a hyperbolic seiferter for $\left(K_{0}, m_{0}\right)$.

Finally we show that $c$ is not $m_{0}$-equivalent to any basic seiferter for $K_{0}=T_{2 n+1, n}$ or a regular fiber in $S^{3}-N\left(K_{0}\right)$, where $m_{0}=n(2 n+1)-1$. If $c$ is $m_{0}$-equivalent to a basic seiferter $s_{2 n+1}$ or $s_{n}$ for $K_{0}$, then $c$ is an exceptional fiber in the lens space $K_{0}\left(m_{0}\right)$. It follows that $K_{0}\left(m_{0}\right)$-int $N(c)$ is a solid torus, a contradiction. Let us suppose that $c$ 
is $m_{0}$-equivalent to $c_{\mu}$. Then, since $\left|\operatorname{lk}\left(c_{\mu}, K_{0}\right)\right|=1$, [9, Proposition 2.22(1)] implies $\operatorname{lk}\left(c, K_{0}\right)= \pm 1+x m_{0}$ for some integer $x$. On the other hand, $\operatorname{lk}\left(c, K_{0}\right)=2 n+2$. We thus have $\pm 1+x m_{0}=2 n+2$, where $n \geq 2$. Then $x=(2 n+1) /\left(2 n^{2}+n-1\right)$ or $(2 n+3) /\left(2 n^{2}+n-1\right)$; these cannot be integers because $2 n^{2}+n-1>2 n+3>2 n+1>0$ for $n \geq 2$. Hence $c$ cannot be $m_{0}$-equivalent to $c_{\mu}$. Let us show that the seiferter $c$ for $\left(K_{0}, m_{0}\right)$ is not $m_{0}$-equivalent to a regular fiber of $S^{3}-N\left(K_{0}\right)$. Since the linking number between $T_{2 n+1, n}$ and a regular fiber of $S^{3}-N\left(T_{2 n+1, n}\right)$ is $\pm n(2 n+1)$. We obtain $\pm n(2 n+1)+x m_{0}=2 n+2$ for some integer $x$ [9, Proposition 2.22(1)], where $n \geq 2$. Then $x=-1+(2 n+1) /\left(2 n^{2}+n-1\right)$ or $1+(2 n+3) /\left(2 n^{2}+n-1\right)$, which cannot be integers for any $n$. Hence $c$ cannot be $m_{0}$-equivalent to a regular fiber in $S^{3}-N\left(K_{0}\right)$.

Proposition 5.5 The lens surgery $\left(T_{2 n-1, n}, n(2 n-1)-1\right)(n \geq 2)$ has a hyperbolic seiferter which is not $(n(2 n-1)-1)$-equivalent to any basic seiferter for $T_{2 n-1, n}$ or a regular fiber of $S^{3}-N\left(T_{2 n-1, n}\right)$.

Proof of Proposition 5.5 In [8, Section 4], we prove that $c^{\prime}$ described in Figure 5.4(1) is a seiferter for the lens surgery $\left(T_{-2 n-3, n+1},(-2 n-3)(n+1)+1\right)$. Twisting $T_{-2 n-3, n+1}$ once along $c^{\prime}$, we obtain Figure 5.4(2). Figure 5.5 demonstrates that the image of $T_{-2 n-3, n+1}$ after the twisting is $T_{2 n-1, n}$; since $1 \mathrm{k}\left(c^{\prime}, T_{-2 n-3, n+1}\right)=2 n+1$, the resulting surgery slope is $(-2 n-3)(n+1)+1+(2 n+1)^{2}=n(2 n-1)-1$. Thus we obtain a lens surgery $\left(T_{2 n-1, n}, n(2 n-1)-1\right)$ for which $c^{\prime}$ remains a seiferter. Note that $\operatorname{lk}\left(c^{\prime}, T_{2 n-1, n}\right)=\operatorname{lk}\left(c^{\prime}, T_{-2 n-3, n+1}\right)=2 n+1$.

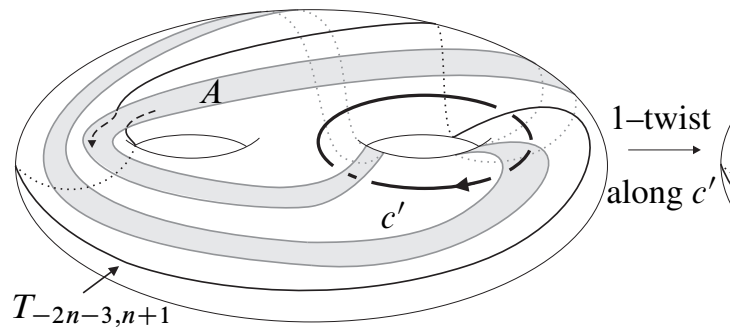

(1)

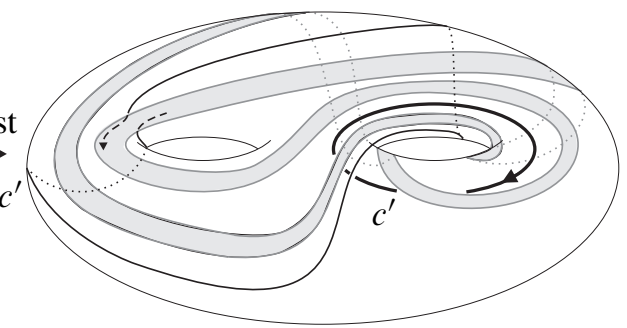

(2)

Figure 5.4: An $n$-Dehn twist is performed along the annulus $A$.

Let $\left(K_{p}, m_{p}\right)$ be the Seifert surgery obtained from $\left(T_{2 n-1, n}, n(2 n-1)-1\right)$ after a $p$-twist along $c^{\prime}$. Then $\left(K_{p}, m_{p}\right)(p \in \mathbb{Z})$ are Berge's lens surgeries on type IV knots. In [8, Section 4] it is shown that each lens space $K_{p}\left(m_{p}\right)$ has a Seifert fibration $\mathcal{F}$ over $S^{2}$ such that $\mathcal{F}$ has two exceptional fibers and $c^{\prime}$ (the image of $c^{\prime}$ after twisting) is a regular fiber of $\mathcal{F}$. Since $1 \mathrm{k}\left(c^{\prime}, T_{2 n-1, n}\right)=2 n+1 \notin\{1, n, 2 n-1\}$, the seiferter $c^{\prime}$ 


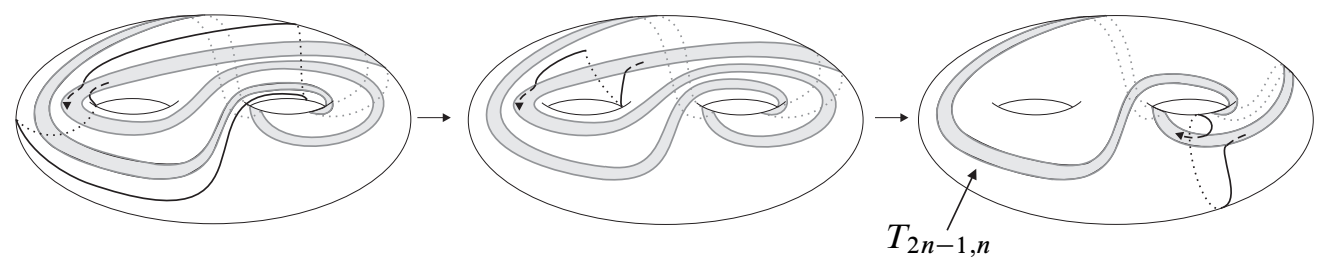

Figure 5.5

is not a basic seiferter for $T_{2 n-1, n}$. Then the argument in the proof of Proposition 5.4 shows that $c^{\prime}$ is a hyperbolic seiferter for the lens surgery $\left(T_{2 n-1, n}, n(2 n-1)-1\right)$, and is not $(n(2 n-1)-1)$-equivalent to a basic seiferter or a regular fiber of $S^{3}-$ $N\left(T_{2 n-1, n}\right)$.

Remark 5.6 The lens surgery $\left(T_{2 n+1, n}, n(2 n+1)-1\right)(n \geq 3)$ has, other than $c$ in Figure 5.2(2), a hyperbolic seiferter which is not $(n(2 n+1)-1)$-equivalent to a basic seiferter or a regular fiber of $S^{3}-N\left(T_{2 n+1, n}\right)$. Let us put $m=n+1$, where $n \geq 2$; then the lens surgery $\left(T_{-2 n-3, n+1},(-2 n-3)(n+1)+1\right)$ in the proof of Proposition 5.5 becomes $\left(T_{-2 m-1, m}, m(-2 m-1)+1\right)$ and $\operatorname{lk}\left(c^{\prime}, T_{-2 m-1, m}\right)=2 m-1$, where $c^{\prime}$ is as in Figure 5.4(1). Let $T_{2 m+1, m} \cup c^{\prime *}$ be the mirror image of the link $T_{-2 m-1, m} \cup c^{\prime}$. Writing $n$ for $m(\geq 3)$, we have the hyperbolic seiferter $c^{\prime *}$ for $\left(T_{2 n+1, n}, n(2 n+1)-1\right)$ which is not $(n(2 n+1)-1)$-equivalent to a basic seiferter or a regular fiber of $S^{3}-N\left(T_{2 n+1, n}\right)$. Since $\left|\operatorname{lk}\left(c^{\prime *}, T_{2 n+1, n}\right)\right|=2 n-1$ is not equal to $\left|\operatorname{lk}\left(c, T_{2 n+1, n}\right)\right|=2 n+2$, the seiferter $c^{\prime *}$ is distinct from $c$.

\section{Band sums and seiferters}

For a 2-component link $k_{1} \cup k_{2}$, we call a band $b$ connecting $k_{1}$ and $k_{2}$ a trivializing band if the band sum $k_{1} \natural_{b} k_{2}$ is a trivial knot in $S^{3}$. Theorem 6.1 below determines when we have a trivializing band connecting a torus knot $T_{p, q}$ and its basic seiferters $s_{p}, s_{q}, c_{\mu}$.

Theorem 6.1 Let $T_{p, q}$ be a nontrivial torus knot with $|p|>q \geq 2$. Then the following hold.

(1) There exists a trivializing band connecting $s_{q}$ and $T_{p, q}$ if and only if $q=2$.

(2) There exists a trivializing band connecting $s_{p}$ and $T_{p, q}$ if and only if $(p, q)=$ $( \pm 3,2)$.

(3) There exists a trivializing band connecting $c_{\mu}$ and $T_{p, q}$ if and only if $(p, q)=$ $( \pm 3,2)$. 
Proof of Theorem 6.1 The band sum of $T_{p, 2}$ and $s_{2}$ described in the first figure of Figure 6.1 is a trivial knot in $S^{3}$. Moreover, if $p=3$, the band sums of $T_{3,2}$ and basic seiferters $s_{3}$ and $c_{\mu}$ described in the second and the third figures of Figure 6.1 are both trivial knots. This fact proves the if parts of Theorem 6.1.

The only if part of assertion (3) is proved by Ishihara and the third author [18]; it is further shown that if a band sum of $c_{\mu}$ and $T_{3,2}$ is a trivial knot, then the band is isotopic to $b_{\mu}$ in Figure 6.1. Thus it is enough to prove the only if parts of (1), (2). The proof is done by relating the band sums to basic seiferters for the degenerate Seifert surgery $\left(T_{p, q}, p q\right)$.
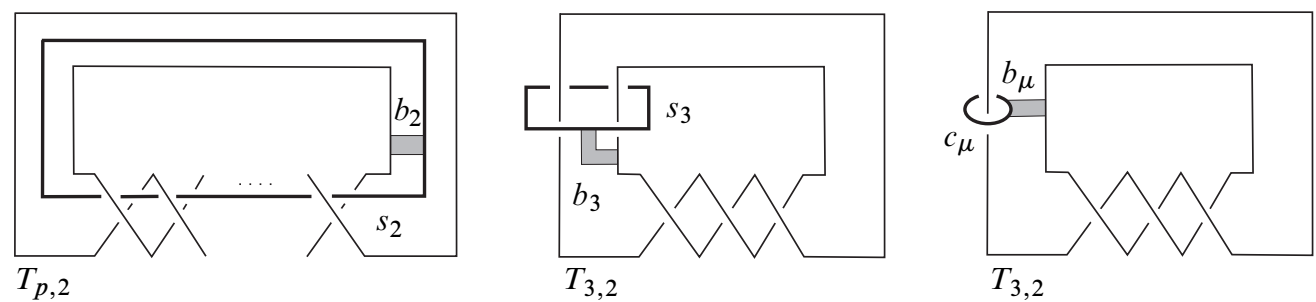

Figure 6.1: Band sums $s_{2} \sharp_{b_{2}} T_{p, 2}, s_{3} \sharp_{b_{3}} T_{3,2}$, and $c_{\mu} \sharp_{b_{\mu}} T_{3,2}$ are trivial knots.

(1) Let $b_{q}$ be a band connecting $s_{q}$ and $T_{p, q}$, and write $k_{q}=s_{q} \bigsqcup_{b_{q}} T_{p, q}$. Take a tubular neighborhood of $T_{p, q}$ so that $N\left(T_{p, q}\right) \cap s_{q}=\varnothing$, and $\partial N\left(T_{p, q}\right) \cap b_{q}$ is an arc. Let $\alpha_{p q}$ be a simple closed curve on $\partial N\left(T_{p, q}\right)$ with slope $p q$ such that $\alpha_{p q} \cap b_{q}=\partial N\left(T_{p, q}\right) \cap b_{q}$. Then, $b_{q}^{\prime}=b_{q}-$ int $N\left(T_{p, q}\right)$ is a band connecting $\alpha_{p q}$ and $s_{q}$ (Figure 6.2). Let $c$ be a knot in $S^{3}-N\left(T_{p, q}\right)$ which is obtained from the band sum $s_{q} \bigsqcup_{b_{q}^{\prime}} \alpha_{p q}$ by pushing away from $\partial N\left(T_{p, q}\right) ; c$ is obtained from the basic seiferter $s_{q}$ by a single $p q$-move using the band $b_{q}^{\prime}$. Note that $c$ is isotopic to $k_{q}$ in $S^{3}$.

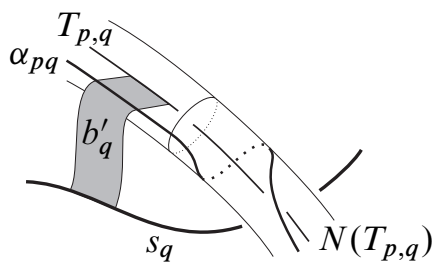

Figure 6.2: Band sum of $s_{q}$ and $T_{p, q}$, and band sum of $s_{q}$ and $\alpha_{p q}$

Now suppose that the band sum $k_{q}$ is a trivial knot in $S^{3}$. Then, $c$ is a seiferter for $\left(T_{p, q}, p q\right)$; moreover, since $c$ is isotopic in $T_{p, q}(p q)$ to the basic seiferter $s_{q}, c$ is 
a nondegenerate exceptional fiber of index $q$ in $T_{p, q}(p q)$. Let $V$ be the solid torus $S^{3}-$ int $N(c)$. We prove the claim below on the position of $T_{p, q}$ in $V$.

Lemma 6.2 The position of $T_{p, q}$ in $V$ is one of the following.

(i) $T_{p, q}$ is a $(q, p)$ cable of $V$.

(ii) $T_{p, q}$ is a $(q, p)$ cable of a $(1, s)$ cable of $V$ for some integer $s$ such that $|s| \geq 2$ and $q=s p \pm 1$.

Proof of Lemma 6.2 Since $c$ is a nondegenerate exceptional fiber in $T_{p, q}(p q) \cong$ $L(p, q) \sharp L(q, p)$, we have the following four possibilities in [9, Corollary 3.21(2), Theorem 3.19(2)(iii)].

(i) $T_{p, q}$ is a $(q, p)$ cable of $V$.

(i') $T_{p, q}$ is a $(p, q)$ cable of $V$.

(ii) $T_{p, q}$ is a $(q, p)$ cable of a $(1, s)$ cable of $V$ for some integer $s$ such that $|s| \geq 2$ and $q=s p \pm 1$.

(ii') $T_{p, q}$ is a $(p, q)$ cable of a $(1, s)$ cable of $V$ for some integer $s$ such that $|s| \geq 2$ and $p=s q \pm 1$.

Since $c$ is isotopic to $s_{q}$ in $T_{p, q}(p q)$, we see $V\left(T_{p, q} ; p q\right)=T_{p, q}(p q)-\operatorname{int} N(c) \cong$ $T_{p, q}(p q)-\operatorname{int} N\left(s_{q}\right)$. This manifold is homeomorphic to $S^{1} \times D^{2} \sharp L(p, q)$ because $T_{p, q}$ is the $(q, p)$ cable of the solid torus $S^{3}-$ int $N\left(s_{q}\right)$. On the other hand, in cases (i') and (ii'), $V\left(T_{p, q} ; p q\right) \cong S^{1} \times D^{2} \sharp L(q, p)$. Thus cases (i') and (ii') do not occur.

In case (i) in Lemma 6.2, $\left|\operatorname{lk}\left(c, T_{p, q}\right)\right|=p$. On the other hand, since $c$ is obtained from $s_{q}$ by a single $p q$-move, we have $\operatorname{lk}\left(c, T_{p, q}\right)=\operatorname{lk}\left(s_{q}, T_{p, q}\right)+\varepsilon p q$, where $c, s_{q}, T_{p, q}$ are oriented adequately and $\varepsilon \in\{ \pm 1\}$ [9, Proposition 2.22(1)]. Hence, $|p+\varepsilon p q|=|p|$. It follows that $q=0, \pm 2$. Since $q \geq 2$, we obtain $q=2$ as claimed in Theorem 6.1.

Now let us consider case (ii) in Lemma 6.2 where $T_{p, q}$ is a $(q, p)$ cable of a $(1, s)$ cable of $V$; then $\operatorname{lk}\left(c, T_{p, q}\right)= \pm p s$. It follows that $|p+\varepsilon p q|=|p s|$ and thus $|1+\varepsilon q|=|s|$. Combining this equality with $|p s-q|=1$ in case (ii), we obtain the inequalities below:

$$
|p s|-1 \leq|p s \varepsilon+1| \leq|1+\varepsilon q|+|p s \varepsilon-\varepsilon q|=|s|+1
$$

It follows $|p s| \leq|s|+2$. Since $|s| \geq 2,|p| \leq 1+\frac{2}{|s|} \leq 2$. This contradicts the assumption $|p|>q \geq 2$. Assertion (1) is thus proved.

(2) Starting with a band sum $k_{p}=s_{p} \bigsqcup_{b_{p}} T_{p, q}$, we follow the argument in (1) with $p$ and $q$ exchanged. Then, we obtain the same statement as in cases (i) and (ii) in 
Lemma 6.2 with $p$ and $q$ exchanged. The modified case (i) then leads to $p=0, \pm 2$. However, this is impossible because $|p|>q \geq 2$. The modified case (ii) leads to the inequality $|q s| \leq|s|+2$, so that $q \leq 1+2 /|s|$. Then, using the fact $|p|>q \geq 2$ and $|s| \geq 2$, we see that $q=2$ and $|s|=2$. Since $|1+\varepsilon q|=|s|$ holds in case (ii), $|1+\varepsilon p|=|s|$ holds in the modified case (ii). We then obtain $p= \pm 3$ and $q=2$, as desired in assertion (2).

Theorem 6.1 implies the following results on seiferters obtained by $m$-moves.

Theorem 6.3 Let $\left(T_{p, q}, m\right)$ be a Seifert surgery on a torus knot $T_{p, q}$ with $|p|>q>2$. Then, there is no seiferter for $\left(T_{p, q}, m\right)$ which is obtained from a basic seiferter by an $m$-move.

Proof of Theorem 6.3 Theorem 6.1 shows that all band sums of $T_{p, q}(|p|>q>2)$ and basic seiferters for $T_{p, q}$ are nontrivial knots in $S^{3}$. Let $\alpha_{m}$ be a simple closed curve in $\partial N\left(T_{p, q}\right)$ with slope $m$. It follows that all band sums of $\alpha_{m}$ and basic seiferters for $T_{p, q}$ are nontrivial knots because $\alpha_{m}$ is isotopic in $N\left(T_{p, q}\right)$ to the core $T_{p, q}$. Thus an arbitrary knot obtained from each basic seiferter for $T_{p, q}$ by an $m$-move is not a seiferter.

Theorem 6.4 Let $\left(T_{p, q}, m\right)$ be a Seifert surgery on a torus knot $T_{p, q}$ with $|p|>q \geq 2$. Suppose that $c$ is a seiferter for $\left(T_{p, q}, m\right)$ which is obtained from a regular fiber of $S^{3}-N\left(T_{p, q}\right)$ by an $m$-move. Then, $c$ is a $(1, m-p q)$ cable of a meridian of $T_{p, q}$, and thus a nonhyperbolic seiferter for $\left(T_{p, q}, m\right)$.

Proof of Theorem 6.4 Suppose that $c$ is a seiferter for $\left(T_{p, q}, m\right)$ which is obtained from a regular fiber $t$ in $S^{3}-N\left(T_{p, q}\right)$ by an $m$-move using a band $b\left(\subset S^{3}-\right.$ int $\left.N\left(T_{p, q}\right)\right) ; c$ is a knot in $S^{3}-N\left(T_{p, q}\right)$ obtained by pushing the band sum $t \natural_{b} \alpha_{m}$ away from $\partial N\left(T_{p, q}\right)$, where $\alpha_{m}$ is a simple closed curve on $\partial N\left(T_{p, q}\right)$ with slope $m$. Our purpose is to show that $c$ is a $(1, m-p q)$ cable of a meridian of $T_{p, q}$.

In the Seifert fibration of $S^{3}-\operatorname{int} N\left(T_{p, q}\right)$, take a regular fiber $\alpha_{p q}$ on $\partial N\left(T_{p, q}\right)$, which represents the slope $p q$. We may assume that there is a small annulus $M$ on $\partial N\left(T_{p, q}\right)$ such that the core curve of $M$ is a meridian of $T_{p, q}$, and that $\alpha_{m}$ and $\alpha_{p q}$ restrict to the same essential arc in the annulus $\partial N\left(T_{p, q}\right)-\operatorname{int} M$.

Now isotope $b$ so that $b \cap \alpha_{m}$ is contained in $\partial N\left(T_{p, q}\right)-$ int $M$, and take the band sum $t \bigsqcup_{b} \alpha_{p q}$. Note that $t \bigsqcup_{b} \alpha_{m}$ and $t \bigsqcup_{b} \alpha_{p q}$ coincide outside of $M$ and are isotopic in $S^{3}$. Let $c_{p q}$ be a knot obtained by pushing $t \natural_{b} \alpha_{p q}$ away from $\partial N\left(T_{p, q}\right)$. Since $c$ is a trivial knot in $S^{3}, c_{p q}$ is also trivial in $S^{3}$. Since $c_{p q}$ is isotopic in $T_{p, q}(p q)$ to 


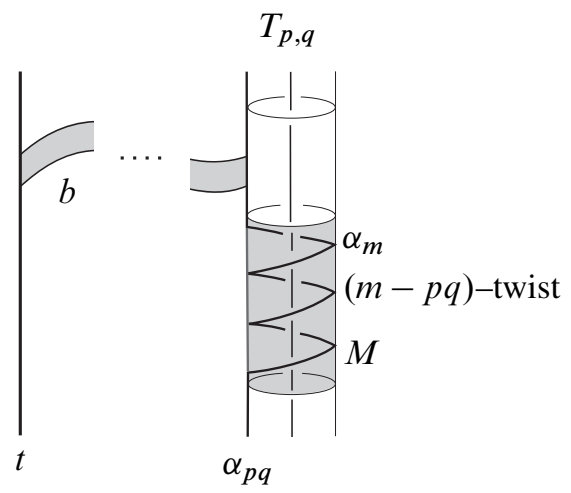

Figure 6.3: A band sum of $\alpha_{m}$ and a regular fiber $t$

the regular fiber $t, c_{p q}$ is a regular fiber in a degenerate Seifert fibration of $T_{p, q}(p q)$. On the other hand, [9, Theorem 3.21(1)] shows that no seiferter for the degenerate Seifert surgery $\left(T_{p, q}, p q\right)$ is a regular fiber. Hence, $c_{p q}$ is not a seiferter. It follows that $c_{p q}$ is an irrelevant seiferter, and so bounds a disk in $S^{3}-T_{p, q}$ (Remark 1.3).

On the position of the band $b$ the following holds.

Lemma 6.5 There exists an annulus $S$ in $S^{3}-\operatorname{int} N\left(T_{p, q}\right)$ such that $\partial S=t \cup \alpha_{p q}$ and $b \subset S$.

Using the annulus $S$ obtained by this lemma, we complete the proof of Theorem 6.4. By an isotopy we may assume further that $S \cap N\left(T_{p, q}\right)=\alpha_{p q}$. Since $S$ contains the band $b, t \bigsqcup_{b} \alpha_{m}$ is the union of the two arcs $\alpha_{m} \cap M$ and $\tau=\partial \overline{(S-b)}-\operatorname{int} M ; \tau$ is isotopic in $S$ with its end points fixed to the arc $\tau^{\prime}$ in Figure 6.4. Note that $\left(\alpha_{m} \cap M\right) \cup \tau^{\prime}$ is a $(1, m-p q)$ cable of a meridian of $T_{p, q}$. This shows that $t \natural_{b} \alpha_{m}$ and thus $c$ is isotopic in $S^{3}-T_{p, q}$ to the $(1, m-p q)$ cable of a meridian of $T_{p, q}$, as claimed.

Proof of Lemma 6.5 Let $A$ be an annulus in $S^{3}-$ int $N\left(T_{p, q}\right)$ with $\partial A=t \cup \alpha_{p q}$. (Since $t$ and $\alpha_{p q}$ are regular fibers in the Seifert fibration of $S^{3}-$ int $N\left(T_{p, q}\right)$, such an annulus is obtained as a union of regular fibers.) Choose orientations of $t$ and $\alpha_{p q}$ which are consistent in $t \bigsqcup_{b} \alpha_{p q}$. We consider two cases according as $t$ and $\alpha_{p q}$ are homologous in $A$ or not. First suppose that $t$ and $\alpha_{p q}$ are homologous in $A$. Then $\operatorname{lk}\left(c_{p q}, T_{p, q}\right)=\operatorname{lk}\left(t, T_{p, q}\right)+\operatorname{lk}\left(\alpha_{p q}, T_{p, q}\right)=2 p q \neq 0$. However, this contradicts the fact that $c_{p q}$ bounds a disk in $S^{3}-T_{p, q}$. Now assume that $t$ and $\alpha_{p q}$ are not homologous in $A$. Then the (adequately oriented) annulus $A$ in $S^{3}-\operatorname{int} N\left(T_{p, q}\right)$ is a Seifert surface for the oriented link $t \cup \alpha_{p q}$. Here, a Seifert surface $F$ for an 

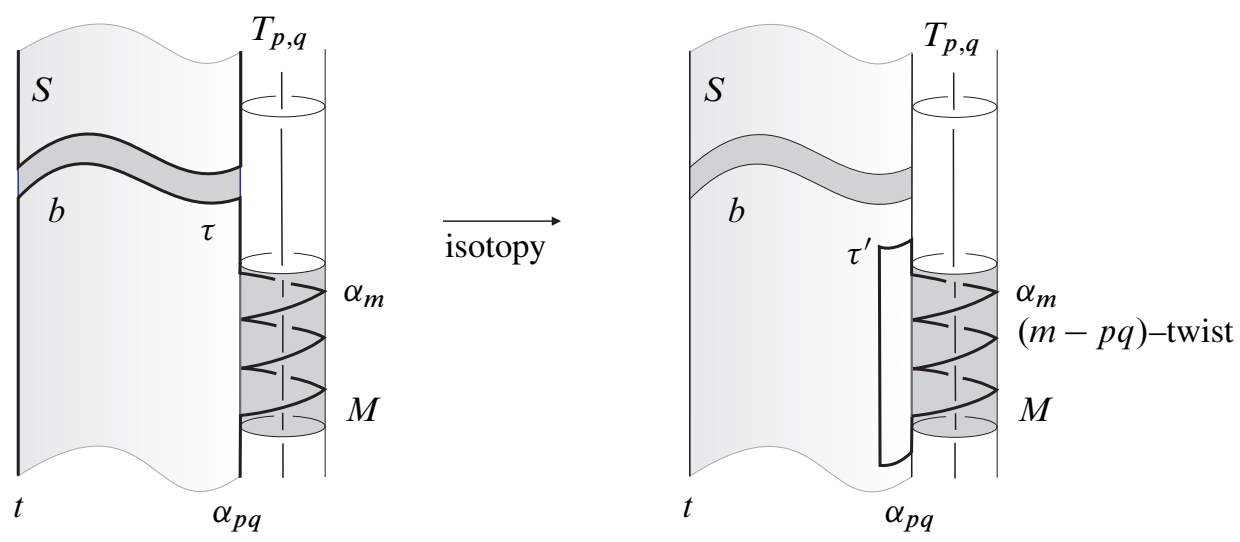

Figure 6.4: $\tau$ is isotopic to $\tau^{\prime}$.

oriented link $L$ is a compact oriented surface such that no component of $F$ is closed and $\partial F=L$. We define $\chi(L)$ to be the maximal Euler characteristic of all Seifert surfaces for $L$. Since $t \natural_{b} \alpha_{p q}$ is a trivial knot in $S^{3}$, we see $\chi\left(t \natural_{b} \alpha_{p q}\right)=1$. Since the oriented link $t \cup \alpha_{p q}$ is nonsplittable, it follows $\chi\left(t \cup \alpha_{p q}\right)=\chi(A)=0$. Then, the minor revision of Hirasawa and Shimokawa's [16, Theorem 1.6] below shows that the oriented link $t \cup \alpha_{p q}$ cobounds an annulus $S$ containing the band $b$, as claimed. By an isotopy we may assume that $S \subset S^{3}-\operatorname{int} N\left(T_{p, q}\right)$.

Theorem 6.6 (A minor revision of [16, Theorem 1.6]) Let $L$ be an oriented link, and $b$ a band connecting (possibly the same) components of $L$ such that $L$ and $b$ induce opposite orientations to their intersection $L \cap b$. Denote the self band sum of $L$ using $b$ by $L_{b}$, an oriented link. Then, $\chi(L) \leq \chi\left(L_{b}\right)-1$ if and only if $L$ has a Seifert surface $S$ such that $\chi(S)=\chi(L)$ and $b \subset S$.

Proof of Theorem 6.6 For a Seifert surface $S\left(\subset M=S^{3}-\operatorname{int} N(L)\right)$ for $L$, consider the three conditions below.

(1) $S$ is taut in $(M, \partial M)$, ie $S$ is incompressible and minimizes the Thurston norm of $[S, \partial S] \in H_{2}(M, N)$, where $N$ is a tubular neighborhood of $\partial S$ in $\partial M$.

(2) $\chi(L)=\chi(S)$

(3) $S$ is a minimal genus Seifert surface for $L$, ie the sum of the genera of the components of $S$ is minimal.

Theorem 1.6 in [16] states that $\chi(L) \leq \chi\left(L_{b}\right)-1$ if and only if $L$ has a minimal genus Seifert surface $S$ such that $b \subset S$. In the proof the authors assume that (3) $\Rightarrow$ (2) and $(1) \Leftrightarrow(3)$ are true. However, these are not true; if a minimal genus Seifert 
surface $S$ for a link $L$ is disconnected, then by tubing two components of $S$, we obtain a minimal genus, compressible Seifert surface $S^{\prime}$ with $\chi\left(S^{\prime}\right)<\chi(L)$. On the other hand, $(1) \Leftrightarrow(2)$ holds by Scharlemann and Thompson [27, Lemma 1.2]. By replacing the word "minimal genus" in the proof of [16, Theorem 1.6] with "taut", we obtain a proof of Theorem 6.6.

Remark 6.7 Among connected Seifert surfaces for a given link, a Seifert surface $S$ has minimal genus if and only if $\chi(S)$ is maximal. Thus, [16, Theorem 1.6] holds for links which have only connected Seifert surfaces. Theorem 6.1(3) is, in fact, proved in [18] by using [16, Theorem 1.6]. However, in the proof [16, Theorem 1.6] is applied only to links with only connected Seifert surfaces.

Corollary 6.8 Let $c$ be a hyperbolic seiferter for $\left(T_{p, q}, m\right)$, where $|p|>q>2$ and $m \neq p q, p q \pm 1$. Then,:

(1) $c$ is $m$-equivalent to a basic seiferter for $T_{p, q}$ (respectively a regular fiber of $S^{3}-N\left(T_{p, q}\right)$ ) if $c$ is an exceptional fiber (respectively a regular fiber) in some Seifert fibration of $T_{p, q}(m)$.

(2) c cannot be obtained from a basic seiferter or a regular fiber of $S^{3}-N\left(T_{p, q}\right)$ by a single $m$-move.

Proof of Corollary 6.8 It follows from the assumption $|p|>q>2$ and $m \neq p q$, $p q \pm 1$ that $T_{p, q}(m)$ is not a connected sum of lens spaces, a lens space, or a prism manifold. Hence, (1) follows from Proposition 2.2, and (2) follows from Theorems 6.3 and 6.4.

\section{References}

[1] K Asano, Homeomorphisms of prism manifolds, Yokohama Math. J. 26 (1978) 19-25 MR524878

[2] R Benedetti, C Petronio, Lectures on hyperbolic geometry, Springer, Berlin (1992) MR1219310

[3] J Berge, Some knots with surgeries yielding lens spaces, unpublished manuscript

[4] M Boileau, J Porti, Geometrization of 3-orbifolds of cyclic type, Astérisque 272 (2001) MR1844891

[5] F Bonahon, J-P Otal, Scindements de Heegaard des espaces lenticulaires, Ann. Sci. École Norm. Sup. 16 (1983) 451-466 MR740078 
[6] A Deruelle, M Eudave-Muñoz, K Miyazaki, K Motegi, Networking Seifert surgeries on knots, IV: Seiferters and branched coverings, from: "Geometry and topology down under”, (C D Hodgson, editor), Amer. Math. Soc. (2013) 235-262

[7] A Deruelle, K Miyazaki, K Motegi, Neighbors of Seifert surgeries on a trefoil knot in the Seifert Surgery Network, preprint

[8] A Deruelle, K Miyazaki, K Motegi, Networking Seifert surgeries on knots, II: The Berge's lens surgeries, Topology Appl. 156 (2009) 1083-1113 MR2493370

[9] A Deruelle, K Miyazaki, K Motegi, Networking Seifert surgeries on knots, Mem. Amer. Math. Soc. 1021, Amer. Math. Soc. (2012) MR2951665

[10] M Eudave-Muñoz, Non-hyperbolic manifolds obtained by Dehn surgery on hyperbolic knots, from: "Geometric topology", (W H Kazez, editor), AMS/IP Stud. Adv. Math. 2, Amer. Math. Soc. (1997) 35-61 MR1470720

[11] M Eudave-Muñoz, On hyperbolic knots with Seifert fibered Dehn surgeries, from: "Proceedings of the First Joint Japan-Mexico Meeting in Topology", Topology Appl. 121 (2002) 119-141 MR1903687

[12] R Fintushel, R J Stern, Constructing lens spaces by surgery on knots, Math. Z. 175 (1980) 33-51 MR595630

[13] C M Gordon, Y-Q Wu, Annular Dehn fillings, Comment. Math. Helv. 75 (2000) 430-456 MR1793797

[14] J E Greene, L-space surgeries, genus bound, and the cabling conjecture, preprint (2012)

[15] A E Hatcher, Notes on basic 3-manifold topology, lecture notes Available at http:// wWw . math . cornell . edu/ hatcher/3M/3Mfds . pdf

[16] M Hirasawa, K Shimokawa, Dehn surgeries on strongly invertible knots which yield lens spaces, Proc. Amer. Math. Soc. 128 (2000) 3445-3451 MR1676336

[17] C Hodgson, J H Rubinstein, Involutions and isotopies of lens spaces, from: "Knot theory and manifolds", (D Rolfsen, editor), Lecture Notes in Math. 1144, Springer, Berlin (1985) 60-96 MR823282

[18] K Ishihara, K Motegi, Band sum operations yielding trivial knots, Bol. Soc. Mat. Mexicana 15 (2009) 103-108 MR2757689

[19] W Jaco, Lectures on three-manifold topology, CBMS Reg. Conf. Series Math. 43, Amer. Math. Soc. (1980) MR565450

[20] B Jiang, S Wang, Y-Q Wu, Homeomorphisms of 3-manifolds and the realization of Nielsen number, Comm. Anal. Geom. 9 (2001) 825-877 MR1868922

[21] K Miyazaki, K Motegi, Seifert fibred manifolds and Dehn surgery, Topology 36 (1997) 579-603 MR1415607 
[22] W D Neumann, F Raymond, Seifert manifolds, plumbing, $\mu$-invariant and orientation reversing maps, from: "Algebraic and geometric topology", (K C Millett, editor), Lecture Notes in Math. 664, Springer, Berlin (1978) 163-196 MR518415

[23] U Oertel, Closed incompressible surfaces in complements of star links, Pacific J. Math. 111 (1984) 209-230 MR732067

[24] P Orlik, Seifert manifolds, Lecture Notes in Mathematics 291, Springer, Berlin (1972) MR0426001

[25] C Petronio, J Porti, Negatively oriented ideal triangulations and a proof of Thurston's hyperbolic Dehn filling theorem, Expo. Math. 18 (2000) 1-35 MR1751141

[26] J H Rubinstein, On 3-manifolds that have finite fundamental group and contain Klein bottles, Trans. Amer. Math. Soc. 251 (1979) 129-137 MR531972

[27] M Scharlemann, A Thompson, Link genus and the Conway moves, Comment. Math. Helv. 64 (1989) 527-535 MR1022995

[28] W P Thurston, The geometry and topology of 3-manifolds, lecture notes, Princeton University (1979) Available at http://library.msri.org/nonmsri/gt3m/

[29] W P Thurston, Three-dimensional manifolds, Kleinian groups and hyperbolic geometry, Bull. Amer. Math. Soc. 6 (1982) 357-381 MR648524

Institute of Natural Sciences, Nihon University

3-25-40 Sakurajosui, Setagaya-ku, Tokyo 156-8550, Japan

Faculty of Engineering, Tokyo Denki University

5 Senju-Asahi-cho, Adachi-ku, Tokyo 120-8551, Japan

Department of Mathematics, Nihon University

3-25-40 Sakurajosui, Setagaya-ku, Tokyo 156-8550, Japan

aderuelle@math.chs.nihon-u.ac.jp, miyazaki@cck.dendai.ac.jp,

motegi@math.chs.nihon-u.ac.jp

http://www.math.chs.nihon-u.ac.jp/ motegi/

Received: 8 November 2012 Revised: 8 November 2013 
